\title{
ALGUNAS PROPIEDADES DE GRÁFICAS GEOMÉTRICAS
}

Tesis que presenta:

Virginia Urrutia Galicia

Para obtener el grado de Doctor en Ciencias

Director: Dr. Eduardo Rivera Campo

Universidad Autónoma Metropolitana-Iztapalapa División de Ciencias Básicas e Ingeniería

Departamento de Matemáticas

Febrero del 2000. 


\section{OAgradecimientos}

OA mis padres de quienes siempre he recibido todo su apoyo y su cariño. Al eldolfo y a Gaby que siempre están en mi corazón.

Quiero agradecer, de manera especial a mi director de tesis, $\$$ Dr. G̊duardo eRivera Campo, por su generosa ayuda y paciencia, a los miembros del jurado:

OMat. Qictor Neumann Lara,

Dra. Hortensia Galeana efánchez,

Dr. Olernando Hurtado Diax,

D). OBernardo Llano Dérex,

Dor sus valiosas observaciones y sugerencias.

Ot la Universidad Otutónoma elletropolitana, Unidad elztapalapa por el apoyo financiero que de ella recibí para realizat mis estudios de doctorado y en especial al Departamento de ellatemáticas.

et mis hermanos, maestros y amigos. 


\title{
Algunas Propiedades de Gráficas Geométricas por
}

\author{
Virginia Urrutia Galicia
}

Universidad Autónoma Metropolitana

Unidad Iztapalapa 


\section{Indice}

- Introducción............................................

- Capítulo 1. Algunas propiedades de $\mathcal{T}(P) \ldots \ldots \ldots \ldots \ldots \ldots \ldots$

1.1 Introducción....................................... 9

1.2 Grados mínimo y máximo .......................... 9

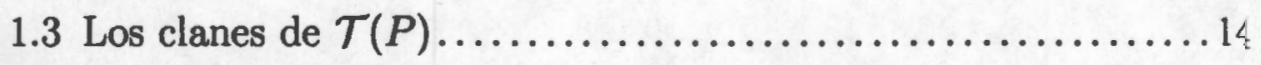

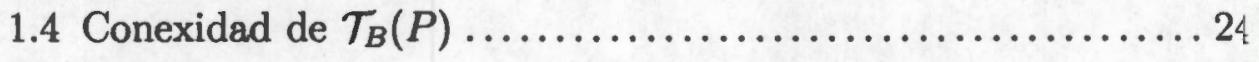

- Capítulo 2. Ciclos Hamiltonianos en la gráfica de trayectorias......30

2.1 Introducción .................................... 30

2.2 Ciclos Hamiltonianos............................... 31

- Capítulo 3. Existencia de árboles generadores planos ............ 46

3.1 Introducción ..................................... 46

3.2 Estrellas y Gusanos ............................... 46

3.3 Triángulos Vacíos y Ciclos de Rectas ................... 55

- Referencias.......................................... 70 


\section{Introducción}

Dada una gráfica abstracta conexa $G$, la gráfica de árboles $T(G)$ de $G$, es la gráfica que tiene un vértice por cada árbol generador de $G \mathrm{y}$ en donde dos árboles son adyacentes si uno puede ser obtenido del otro mediante un solo intercambio de aristas. En [5] R.L. Cummings demostró que $T(G)$ es una gráfica hamiltoniana (en [10] H. Shank da una prueba más corta). Daremos ahora una versión geométrica de este problema.

Sea $P$ una colección de $n$ puntos en el plano en posición general, es decir, cualesquiera tres puntos de $P$ no son colineales.

Una gráfica geométrica $G$ sobre $P$ es una gráfica cuyo conjunto de vértices es $P$ y tal que toda arista de $G$ es un segmento de recta que une dos puntos de $P$. En particular la gráfica geométrica completa $K(P)$ de $P$, es la gráfica greométrica que contiene todas las aristas posibles entre puntos de $P$. (Véase figura 1).

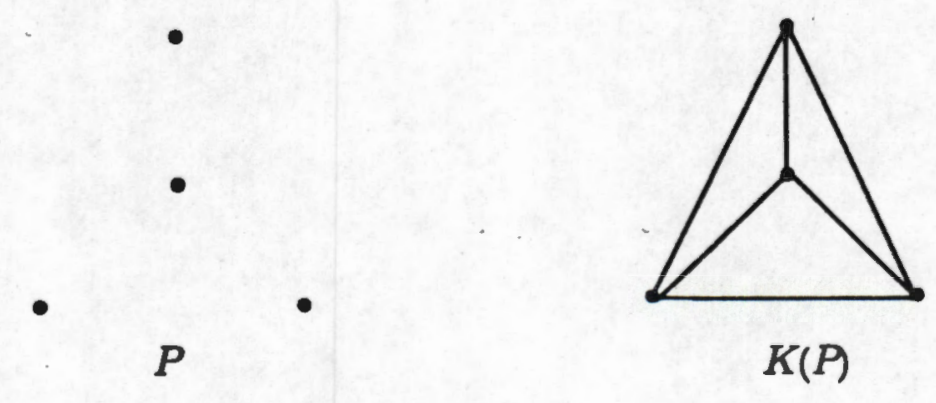

Figura 1

Un árbol generador plano sobre $P$, es un árbol generador de $K(P)$, tal que cualesquiera dos aristas se intersectan a lo más en alguno de sus extremos. 
La gráfica de árboles $\mathcal{T}(P)$ de $P$, es la gráfica que tiene un vértice por cada árbol generador plano de $K(P)$ y dos árboles generadores planos $R$ y $S$ de $K(P)$ son adyacentes en $\mathcal{T}(P)$ si $R$ puede ser obtenido de $S$ reemplazando una arista $s$ de $S$ por una arista $r$ de $R$, es decir $R=(S+r)-s$ con $r \in E(R)$ y $s \in E(S)$. En el ejemplo de la figura $2, R$ y $S$ son dos árboles adyacentes de $\mathcal{T}(P)$.
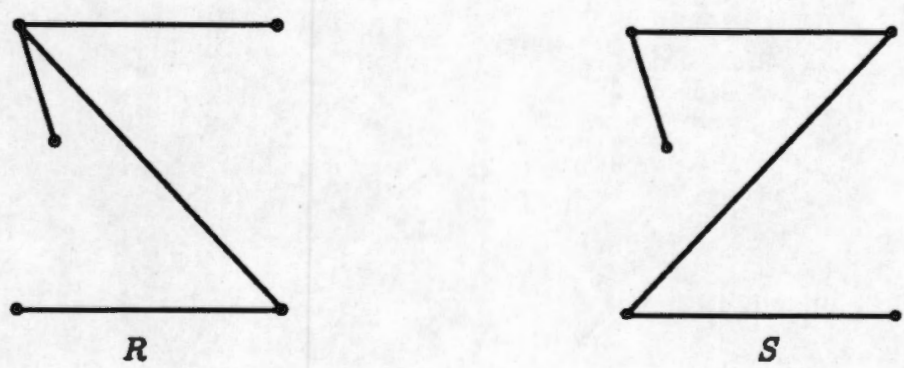

Figura 2

Un problema, aún sin resolver es el de determinar si $\mathcal{T}(P)$ es siempre una gráfica hamiltoniana, sin embargo D. Avis y K. Fukuda demostraron en [2] que $\mathcal{T}(P)$ es conexa y C. Hernando et al, demostraron en [7] que en el caso en que $P$ es una colección de puntos en posición convexa (esto es los puntos de $P$ forman los vértices de un polígono convexo), $\mathcal{T}(P)$ es una gráfica hamiltoniana.

Otras versiones geométricas de dicho problema han sido formuladas, todas ellas definidas de manera análoga. Por ejemplo, considere, en lugar de la gráfica de árboles, a la gráfica de triangulaciones o a la gráfica de trayectorias o bien a la gráfica de las $k$-gráficas conexas (una $k$-gráfica conexa es una gráfica geométrica, plana, conexa y con $k$ aristas), etc. El problema en cada 
caso es determinar si la gráfica dada es hamiltoniana y encontrar algunas propiedades de dichas gráficas.

En el capítulo 1, estudiaremos algunas propiedades de la gráfica de árboles $\mathcal{T}(P)$ de $P$ y demostraremos que la subgráfica $\mathcal{T}_{B}(P)$ de $\mathcal{T}(P)$ es conexa, donde $B$ es un bosque generador plano de $K(P)$ y $\mathcal{T}_{B}(P)$ es la gráfica en la que cada vértice es un árbol generador plano de $K(P)$ que contiene todas las aristas de $B$. Dos árboles son adyacentes en $\mathcal{T}_{B}(P)$ si uno puede ser obtenido del otro mediante un sólo intercambio de aristas. En la figura 3 se muestra un ejemplo.

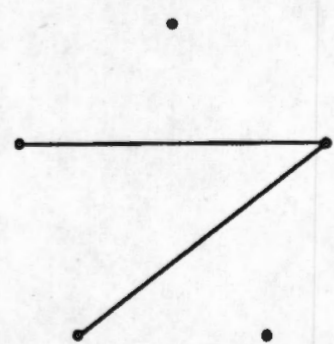

$B$

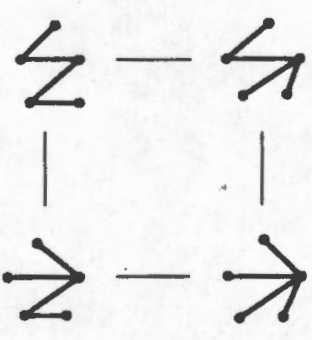

$$
\mathcal{T}_{\mathrm{B}}(P)
$$

Figura 3

En el capítulo 2, demostraremos que si $P$ es una colección de puntos en posición convexa, entonces la subgráfica $T(P)$ de $\mathcal{T}(P)$, inducida por el conjunto de trayectorias generadoras planas de $K(P)$, es hamiltoniana. $\mathrm{Si}$ $|P|=3$ y $|P|=4$, entonces $T(P)$ es mostrada en la figura 4.

Finalmente, en el capítulo 3, daremos algunos resultados relacionados con la existencia de árboles generadores planos en gráficas geométricas. 

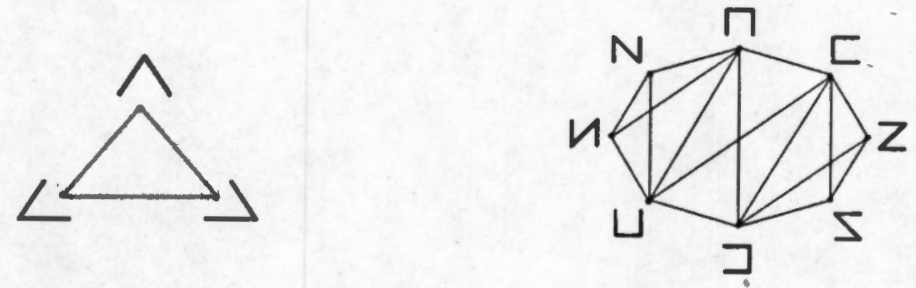

Figura 4

Daremos a continuación algunas definiciones básicas que serán usadas en los siguientes capítulos.

Una gráfica $G$ consiste de un conjunto finito no-vacío $V=V(G)$ de puntos llamados vértices junto con conjunto $E=E(G)$ de pares no ordenados de vértices de $V$, llamadas aristas.

Si $G$ es una gráfica entonces $G^{c}$ es la gráfica tal que $V\left(G^{c}\right)=V(G)$ y dos vértices son adyacentes en $G^{c}$ si y sólo si no son adyacentes en $G$.

Una subgráfica $H$ de $G$ es una gráfica que tiene todos sus vértices y sus aristas en $G$.

Una subgráfica generadora de $G$ es una subgráfica que contiene todos los vértices de $G$.

Una subgráfica inducida $H$ de $G$ es una subgráfica de $G$ tal que dos vértices son adyacentes en $H$ si y sólo si son adyacentes en $G$.

Si $G$ es una gráfica y e es una arista de $G$, entonces $G$ - e denotará a la gráfica obtenida de $G$ al quitarle la arista e y si $f$ no es una arista de $G$, pcro tiene ambos extremos en $V(G)$ entonces $G+f$ denotará a la gráfica obtenida al agregarle a $G$ la arista $f$. 
El grado $\operatorname{gr}_{G}(v)$ de $v$ en $G$ es el número de vértices adyacentes a $v$.

Si $\operatorname{gr}_{G}(v)=1$, entonces decimos que $v$ es un vértice terminal de $G$. Si e es una arista que tiene como uno de sus extremos a un vértice terminal, entonces decimos que $e$ es una arista terminal.

Definimos

$$
\begin{aligned}
\delta(G) & :=\min \left\{\operatorname{gr}_{G}(v) \mid v \in V(G)\right\} \\
\Delta(G) & :=\operatorname{máx}\left\{\operatorname{gr}_{G}(v) \mid v \in V(G)\right\}
\end{aligned}
$$

como el grado mínimo y el grado máximo de $G$, respectivamente.

Una gráfica $G$ es conexa si cualquier par de vértices está unido por una trayectoria de $G$.

Una subgráfica de $G$ conexa y maximal es llamada componente conexa de $G$.

Un bosque es una gráfica acíclica.

Un árbol es una gráfica acíclica y conexa.

Una trayectoria es un árbol con exactamente dos vértices terminales.

Una estrella es un árbol con exactamente un vértice no-terminal.

Un uniciclo es una gráfica que contiene un único ciclo.

Un clan de una gráfica $G$, es una subgráfica completa maximal de $G$. 
El número de clan $\omega(G)$ de una gráfica $G$, es el número de vértices en $\mathrm{cl}$ clan de cardinalidad máxima de $G$.

Si una gráfica $G$ contiene un ciclo generador $C$ entonces decimos que $G$ es hamiltoniana y que $C$ es un ciclo hamiltoniano de $G$.

Si $T$ es una trayectoria generadora de una gráfica $G$ entonces $T$ es llamada una trayectoria hamiltoniana de $G$.

Un conjunto $A$ de puntos en el plano es convexo, si para cualesquiera dos puntos $x, y$ de $A$ el segmento $\overline{x y}$ está totalmente contenido en $A$.

Si $P$ es una colección de puntos en el plano en posición general, definimos la cerradura convexa, $C H(P)$, de $P$ como la intersección de todos los conjuntos convexos en el plano que contienen a $P$. Si $p \in P$ no está en la frontera de $C H(P)$ entonces a $p$ lo llamaremos un punto interior de $P$.

Si $G$ es una gráfica geométrica sobre $P$ tal que cualquier par de aristas se intersecta a lo más en alguno de sus extremos, entonces decimos que $G$ es una gráfica geométrica plana sobre $P$.

Si $D$ es una gráfica geométrica plana sobre $P$ tal que todas sus caras tienen exactamente 3 lados, excepto posiblemente la cara exterior, entonces decimos que $D$ es una triangulación geométrica. Una triangulación geométrica $D$ es maximal si $D+e$ no es plana, para cualquier $e \in E(K(P))-E(D)$. A lo largo de este trabajo, a una triangulación geométrica maximal la llamaremos simplemente una triangulación. 


\section{Capítulo 1}

\section{$1 \quad$ Algunas Propiedades de $\mathcal{T}(P)$}

\subsection{Introducción}

Sean $P$ una colección de $n$ puntos en el plano en posición general y $\mathcal{T}(P)$ la gráfica de árboles de $P$.

En este capítulo daremos una cota inferior para el grado mínimo de $\mathcal{T}(P)$ y una cota superior para el grado máximo de $\mathcal{T}(P)$. C. Hernando et al en [7] dieron cotas para el grado mínimo y máximo de $\mathcal{T}(P)$ en el caso en que $P$ es una colección de puntos en posición convexa. Caracterizaremos además los clanes de $\mathcal{T}(P)$ y encontraremos una cota inferior y otra superior para el número de clan. Por último demostraremos que si $B$ es un bosque generador plano sobre $P$ y $\mathcal{T}_{B}(P)$ es la gráfica con un vértice por cada árbol generador plano de $K(P)$ que contiene a $B$ y en donde dos árboles son adyacentes si uno puede ser obtenido del otro mediante un solo intercambio de aristas que no estén en $B$, entonces $\mathcal{T}_{B}(P)$ es un gráfica conexa.

\subsection{Grados mínimo y máximo en $\mathcal{T}(P)$.}

Sea $S$ un árbol generador plano de $K(P)$. Para toda arista e $\in E(K(P)) \backslash E(S)$ existe un único ciclo $S_{e}$ de $K(P)$ contenido en $S+e$. Claramente $(S+e)-x$ es un árbol generador de $K(P)$, para cada arista $x$ de $S_{e}$. Si $(S+e)-x$ es plano y $e \neq x$, entonces $(S+e)-x$ es un árbol generador plano de $\mathcal{T}(P)$, el cual es adyacente a $S$. 
Teorema 1.2.1 $\delta(\mathcal{T}(P)) \geq 2(n+k-2)$ para cualquier conjunto $P$ de $n \geq 2$ puntos en posición general en el plano, con $k$ puntos interiores.

\section{DEMOSTRACIÓN}

Sea $S$ un árbol generador plano de $K(P)$ y $D$ una triangulación de $P$ que incluya todas las aristas de $S$. Sea $e \in E(D) \backslash E(S)$. Como $S+e$ es una subgráfica plana de $K(P)$, entonces para cada arista $e^{\prime} \neq e, e^{\prime} \in S_{e}$, $(S+e)-e^{\prime}$ es un árbol generador plano de $K(P)$ el cual es adyacente a $S$ en $\mathcal{T}(P)$, por lo tanto

$$
\underset{\mathcal{T}(P)}{\operatorname{gr}}(S) \geq \sum_{e \in E(D) \backslash E(S)}\left|E\left(S_{e}\right)\right|-1 \geq 2[|E(D)|-|E(S)|]
$$

Como $D$ es una triangulación geométrica y $n-k$ es el número de puntos en la frontera de $C H(P)$ entonces $|E(D)|=3 n-6-(n-k-3)=2 n+k-3$.

Por lo tanto

$$
\underset{\mathcal{T}(P)}{\operatorname{gr}}(S) \geq 2[2 n+k-3-(n-1)]=2[n+k-2] .
$$

Corolario 1.2.2 (Hernando C.,et.al. (7)) $\delta(\mathcal{T}(P)) \geq 2(n-2)$, para cualquier conjunto $P$ de $n \geq 2$ puntos en el plano en posición convexa.

Para ver que la cota es justa consideremos la estrella $S$ sobre $n$ puntos, de los cuales $k$ son puntos interiores, colocados como se muestra en la figura 1.1. No es difícil ver que $\underset{\tau(P)}{\operatorname{gr}(S)}=2[n+k-2]$ y por lo tanto la cota es justa. 


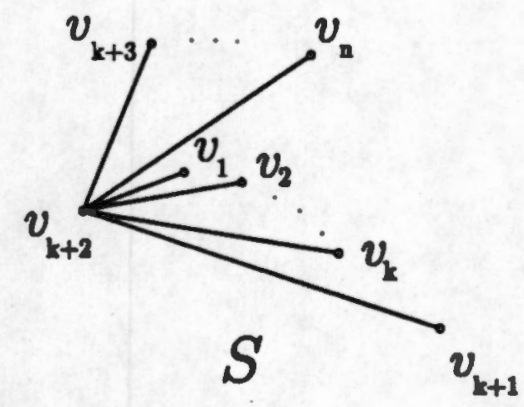

Figura 1.1

Encontraremos ahora una cota superior para el grado máximo en $\mathcal{T}(P)$. Para ésto, daremos las siguientes definiciones y demostraremos los siguientes dos lemas.

Si $S$ es un árbol generador plano de $K(P)$ y $u$ es un vértice terminal de $S$, definimos $E_{1}$ como el conjunto de aristas en $E(K(P)) \backslash E(S)$ incidentes con $u$ y $E_{2}$ como el conjunto de aristas en $E(K(P)) \backslash E(S)$ no incidentes con $u$. Ver figura 1.2 .

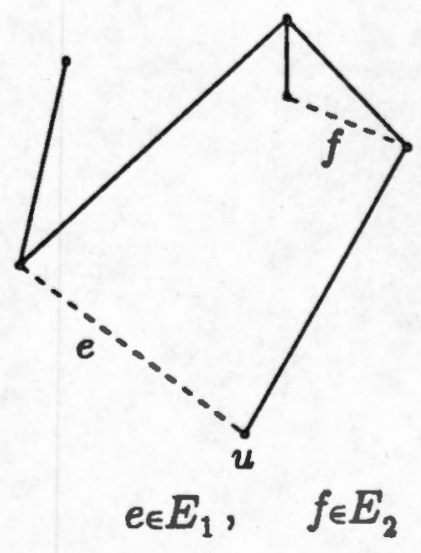

Figura 1.2 
Lema 1.2.3 $\sum_{e \in E_{1}}\left|E\left(S_{e}\right)\right| \leq \frac{(n-2)(n+3)}{2}$.

\section{Demostración}

Si $n=3$, entonces $S$ es una trayectoria $u, v, w$ de longitud $3, e=u w$ es la única arista de $E_{1}$ y $\left|E\left(S_{e}\right)\right|=3$.

Supongamos el resultado válido para $n=k$ y sea $n=k+1$. Sea $v \neq u$ un vértice terminal de $S$, como $S^{\prime}=S-v$ es un árbol generador plano de $K\left(P^{\prime}\right)$ y $K\left(P^{\prime}\right)=K(P \backslash\{v\})$ entonces, por hipótesis de inducción

$$
\sum_{e \in E_{1}^{\prime}}\left|E\left(S_{e}^{\prime}\right)\right| \leq \frac{(k-2)(k+3)}{2}
$$

donde $E_{1}^{\prime}=E_{1} \backslash\{u, v\}$ es el conjunto de aristas en $E\left(K\left(P^{\prime}\right)\right) \backslash E\left(S^{\prime}\right)$ las cuales son incidentes con $u$.

Como $\left|E\left(S_{\text {uv }}\right)\right| \leq n=k+1$ entonces

$$
\begin{aligned}
\sum_{e \in E_{1}}\left|E\left(S_{e}\right)\right|= & \sum_{e \in E_{1}^{\prime}}\left|E\left(S_{e}^{\prime}\right)\right|+\left|E\left(S_{u v}\right)\right| \\
& \leq \frac{(k-2)(k+3)}{2}+k+1 \\
& =\frac{(k-1)(k+4)}{2}=\frac{(n-2)(n+3)}{2}
\end{aligned}
$$

Lema 1.2.4

$$
\sum_{e \in E(K(P)) \backslash E(S)}\left|E\left(S_{e}\right)\right| \leq \frac{(n-1)(n-2)(n+6)}{6} .
$$




\section{DEMOSTRAClón}

Si $n=3$, entonces $S$ es una trayectoria $u, v, w$ de longitud $3, e=u w$ es la única arista de $E(K(P)) \backslash E(S)$ y $\left|E\left(S_{e}\right)\right|=3$. Supongamos el resultado válido para $n=k$ y sea $n=k+1$.

Sea $P^{\prime \prime}=P \backslash\{u\}$ y $S^{\prime \prime}=S-u$. Como $S^{\prime \prime}$ es un árbol generador plano de $K\left(P^{\prime \prime}\right)$ y $\left|P^{\prime \prime}\right|=k$ entonces

$$
\sum_{e \in E\left(K\left(P^{\prime \prime}\right)\right) \backslash E\left(S^{\prime \prime}\right)}\left|E\left(S_{e}^{\prime \prime}\right)\right| \leq \frac{(k-1)(k-2)(k+6)}{6}
$$

Como $E\left(K\left(P^{\prime \prime}\right)\right) \backslash E\left(S^{\prime \prime}\right)=E_{2}$ entonces

$$
\begin{aligned}
\sum_{e \in E(K(P)) \backslash E(S)}\left|E\left(S_{e}\right)\right| & =\sum_{e \in E_{1}}\left|E\left(S_{e}\right)\right|+\sum_{e \in E_{2}}\left|E\left(S_{e}\right)\right| \\
& =\sum_{e \in E_{1}}\left|E\left(S_{e}\right)\right|+\sum_{e \in E\left(K\left(P^{\prime \prime}\right)\right) \backslash E\left(S^{\prime \prime}\right)}\left|E\left(S_{e}^{\prime \prime}\right)\right| \\
& \leq \frac{(n-2)(n-3)}{2}+\frac{(k-1)(k-2)(k+6)}{6} \\
& =\frac{(n-2)(n+3)}{2}+\frac{(n-2)(n-3)(n+5)}{6} \\
& =\frac{(n-1)(n-2)(n+6)}{6}
\end{aligned}
$$

Teorema 1.2.5 $\Delta(\mathcal{T}(P)) \leq \frac{(n-1)(n-2)(n+3)}{6}$ para cualquier conjunto $P$ con $n \geq 2$ puntos en posición general en $\mathbb{R}^{2}$.

\section{DEMOSTRACIÓN}

Si $S$ es un árbol generador plano de $K(P)$, entonces por lema 1.2.4,

$$
\underset{T(P)}{\operatorname{gr}}(S) \leq \sum_{e \in E(K(P)) \backslash E(S)}\left|E\left(S_{e}\right)\right|-1
$$




$$
\begin{aligned}
& =\sum_{e \in E(K(P)) \backslash E(S)}\left|E\left(S_{e}\right)\right|-\left[\left(\begin{array}{l}
n \\
2
\end{array}\right)-(n-1)\right] \\
& \leq\left[\frac{(n-1)(n-2)(n+6)}{6}\right]-\left[\left(\begin{array}{l}
n \\
2
\end{array}\right)-(n-1)\right] \\
& =\frac{(n-1)(n-2)(n+3)}{6}
\end{aligned}
$$

Para mostrar que esta cota es justa, consideremos el conjunto $P=$ $\left\{v_{1}, \ldots, v_{n}\right\}$, con $k$ puntos interiores y $S$ la trayectoria generadora plana de $K(P)$ que tiene como conjunto de aristas a $E(S)=\left\{v_{1}, v_{2}, v_{2}, v_{3}, \ldots, v_{n-1} v_{n}\right\}$ (véase la figura 1.3). En este caso $S+e$ es una subgráfica plana de $K(P)$, para toda $e \in E(K(P))\left(E(S)\right.$ y $S$ es adyacente a exactamente $\frac{(n-1)(n-2)(n+3)}{6}$ árboles generadores planos de $\mathcal{T}(P)$.

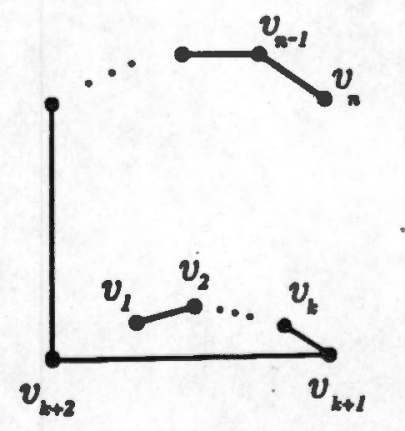

Figura 1.3

\subsection{Los clanes de $\mathcal{T}(P)$.}

En esta sección caracterizaremos los clanes de $\mathcal{T}(P)$ y daremos cotas para el tamaño de sus clanes. 
Sea $U$ un uniciclo generador plano de $K(P)$ y $C$ el único ciclo de $U$. Para cada arista $e$ de $C$, la gráfica $U-e$ es un árbol generador plano de $K(P)$. Además si $e$ y $e^{\prime}$ son dos aristas de $C$, entonces $U-e$ y $U-e^{\prime}$ son vértices adyacentes en $\mathcal{T}(P)$. Ver ejemplo en la figura 1.4.
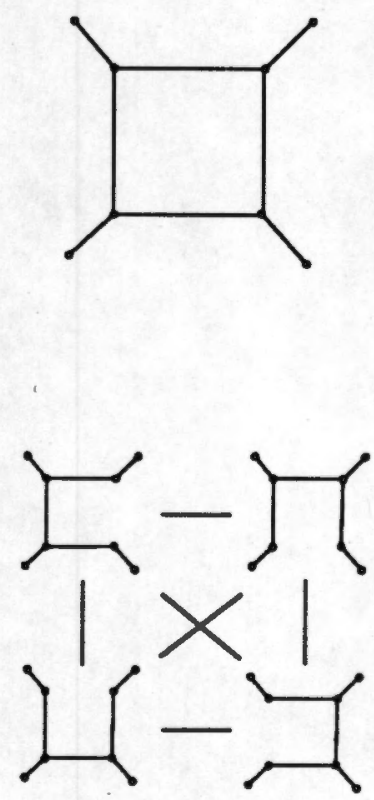

Figura 1.4

Teorema 1.3.1 La subgráfica $U_{C}$ de $\mathcal{T}(P)$, inducida por el conjunto de vértices $\{U-e \mid e \in C\}$ es un clan de $\mathcal{T}(P)$.

\section{DEMOSTRACIÓN}

Como $U-e$ y $U-e^{\prime}$ son adyacentes en $\mathcal{T}(P)$ para cualesquiera dos aristas $e, e^{\prime}$ de $C$, entonces $U_{C}$ es una subgráfica completa. 
Sean $e_{1}, e_{2}, \ldots, e_{m}$ las aristas de $C$ y para $i=1,2, \ldots, m$ denotamos por $U_{i}$ al árbol generador plano $U-e_{i}$. Sea $R$ un árbol generador plano de $K(P)$ adyacente o igual a $U_{i}$, para toda $i=1,2, \ldots, m$. Sea $e$ una arista de $U$ que no esté en $C$. Si $e \notin R$ entonces $E\left(U_{i}\right) \backslash\{e\} \subset E(R)$ para $i=1,2, \ldots, m$, ya que $e \in E\left(U_{i}\right)$ y $R$ es adyacente a $U_{i}$. En particular $\left\{e_{1}, e_{2}, \ldots, e_{m}\right\} \subset E(R)$, lo cual no es posible ya que $R$ no contiene ciclos, por lo tanto $E(U) \backslash E(C) \subset E(R)$.

Como $R$ no contiene ciclos, entonces $e_{i} \notin E(R)$ para alguna $i \in\{1,2, \ldots, m\}$. Sean $j, k \in\{1,2, \ldots, m\}$ distintas de $i$. Como $R$ es adyacente a $U_{j}$ y a $U_{k}$ en $\mathcal{T}(P)$, y $e_{i}$ es una arista de $U_{j}$ y de $U_{k}$, entonces $E\left(U_{j}\right) \backslash\left\{e_{i}\right\} \subset E(R)$ y $E\left(U_{k}\right) \backslash\left\{e_{i}\right\} \subset E(R)$; en particular $E(C) \backslash\left\{e_{i}\right\} \subset E(R)$. Esto junto con que $E(U) \backslash E(C) \subset E(R)$ implican que $R=U_{i}$ y por lo tanto $U_{C}$ es un clan de $\mathcal{T}(P)$.

Sea $B$ un bosque generador plano de $K(P)$ con exactamente dos componentes conexas y sea $M$ el conjunto de aristas $e$ de $K(P)$ tal que $B+e$ es un árbol generador plano de $K(P)$.

Para cualesquiera dos aristas $e, e^{\prime}$ de $M$, los árboles $B+e$ y $B+e^{\prime}$ son adyacentes en $\mathcal{T}(P)$. Ver figura 1.5.

Teorema 1.3.2 Si $|M| \geq 3$, entonces la subgráfica $B_{M}$ de $\mathcal{T}(P)$, inducida por $\{B+e \mid e \in M\}$ es un clan de $\mathcal{T}(P)$. 


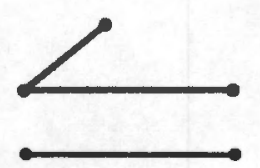

$B$

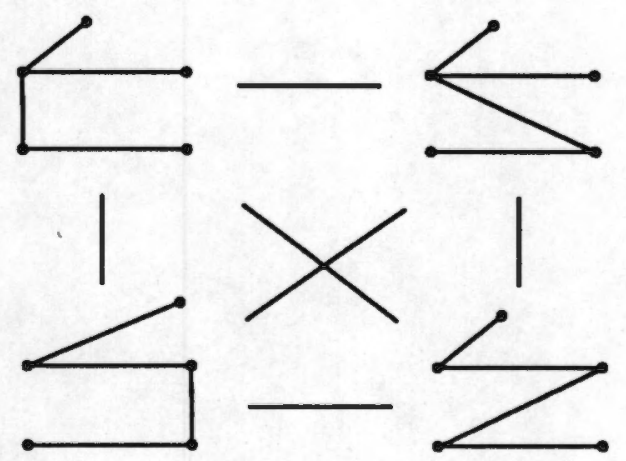

Figura 1.5

\section{DEMOSTRACIÓN}

Como $B+e$ y $B+e^{\prime}$ son adyacentes en $\mathcal{T}(P)$ para cualesquiera dos aristas $e, e^{\prime}$ de $M$, entonces $B_{M}$ es una subgráfica completa de $\mathcal{T}(P)$.

Sean $e_{1}, e_{2}, \ldots, e_{m}$ las aristas de $M$. Para $i=1,2, \ldots, m$, denotamos por $B_{i}$ al árbol $B+e_{i}$. Sea $S$ un árbol generador plano de $K(P)$ adyacente o igual a $B_{i}$ en $\mathcal{T}(P)$ para toda $i=1,2, \ldots, m$.

Supongamos que $M \subset E(S) ;$ como $e_{2}, \ldots, e_{m} \notin E\left(B_{1}\right)$ entonces $\left\{e_{2}, e_{3}, \ldots, e_{m}\right\}$ $\subset E(S) \backslash E\left(B_{1}\right)$, lo cual no es posible, ya que $m \geq 3$ y $S$ es adyacente a $B_{1}$ en $\mathcal{T}(P)$.

Por lo tanto $e_{k} \notin E(S)$ para alguna $k=1,2, \ldots, m$. Como $S$ es adyacente a $B_{k}$ en $\mathcal{T}(P)$, entonces $E(B)=E\left(B_{k}\right)-\left\{e_{k}\right\} \subset E(S)$ y como $B$ es un bosque 
generador plano de $K(P)$ con exactamente dos componentes conexas y $S$ es un árbol generador plano de $K(P)$, entonces $S=B+e^{\prime}$, para alguna arista $e^{\prime} \in M$. Esto implica que $e^{\prime}=e_{i}$ y $S=B_{i}$, para alguna $i \neq k$. Por lo tanto $B_{M}$ es un clan de $\mathcal{T}(P)$.

Teorema 1.3.3 Si $\Omega$ es un clan de $\mathcal{T}(P)$, entonces exactämente una de las siguientes condiciones se cumple.

a) existe un uniciclo generador plano $U$ de $K(P)$ con $C$ su único ciclo tal $q u e \Omega=U_{C}$.

b) existe un bosque generador plano $B$ de $K(P)$ con exactamente dos componentes conexas tal que $\Omega=B_{M}$, donde $M$ es el conjunto de aristas e de $K(P)$ tales que $B+e$ es un árbol generador plano de $K(P)$.

\section{DEMOSTRACIÓN}

Sea $\Omega=\left\{R_{1}, R_{2}, \ldots, R_{m}\right\}$ un clan de $\mathcal{T}(P)$ y sean $e_{1} \in E\left(R_{1}\right)$ y $e_{2} \in$ $E\left(R_{2}\right)$ tales que $R_{1}=\left(R_{2}-e_{2}\right)+e_{1}$.

Supongamos que $e_{1} \in R_{k}$ y $e_{2} \notin R_{k}$ para alguna $k=3,4, \ldots, m$. Como $R_{k}$ es adyacente a $R_{2}$ en $\mathcal{T}(P)$, entonces $E\left(R_{2}\right) \backslash\left\{e_{2}\right\} \subset E\left(R_{k}\right)$ y por lo tanto $R_{k}=\left(R_{2}-e_{2}\right)+e_{1}=R_{1}$ lo cual no es posible; por lo tanto si $e_{1} \in R_{k}$, entonces $e_{2} \in R_{k}$. De manera análoga si $e_{2} \in E\left(R_{k}\right)$ para alguna $k=3,4, \ldots, m$, entonces $e_{1} \in E\left(R_{k}\right)$.

Como $R_{3}, R_{4}, \ldots, R_{m}$ son adyacentes por pares en $\mathcal{T}(P)$, entonces $e_{1}, e_{2} \in$ $E\left(R_{k}\right)$ para toda $k=3,4, \ldots, m$ ó $e_{1}, e_{2} \notin E\left(R_{k}\right)$ para toda $k=3,4, \ldots, m$. 
CASO 1. $e_{1}, e_{2} \in E\left(R_{k}\right)$ para toda $k=3,4, \ldots, m$.

Sea $C$ el único ciclo contenido en el uniciclo $U=R_{1}+e_{2}=R_{2}+e_{1}$ y sea $3 \leq k \leq m$. Como $R_{k}$ no contiene ciclos entonces existe una arista $e \in E(C)$ la cual no es arista de $R_{k}$. Como e es una arista de $R_{1}$ y $R_{k}$ es adyacente a $R_{1}$ en $\mathcal{T}(P)$, entonces $E\left(R_{1}\right) \backslash\{e\} \subset E\left(R_{k}\right)$; de manera análoga $E\left(R_{2}\right) \backslash\{e\} \subset E\left(R_{k}\right)$.

Por lo tanto $\left(E\left(R_{1}\right) \cup E\left(R_{2}\right)\right) \backslash\{e\} \subset E\left(R_{k}\right)$ y como $E(U)=E\left(R_{1}\right) \cup$ $E\left(R_{2}\right)$, entonces $R_{k}=U-e \in U_{C}$. Como $R_{1}, R_{2} \in U_{C}$, entonces $\Omega=U_{C}$.

CAso 2. $e_{1}, e_{2}, \notin E\left(R_{k}\right)$ para toda $k=3,4, \ldots, m$.

Claramente $B=R_{1}-e_{1}=R_{2}-e_{2}$ es un bosque generador plano de $K(P)$ con exactamente dos componentes conexas. Sea $3 \leq k \leq m$ y $M$ el conjunto de aristas $e$ de $K(P)$ tal que $B+e$ es un árbol generador plano de $K(P)$. Como $e_{1} \in E\left(R_{1}\right)$ y $R_{k}$ es adyacente a $R_{1}$ en $T(P)$, entonces $E(B)=E\left(R_{1}\right) \backslash\left\{e_{1}\right\} \subset E\left(R_{k}\right)$ y como $R_{k}$ es un árbol plano entonces existe una arista $e_{k} \in M$ tal que $R_{k}=B+e_{k}$. Claramente $R_{1}=B+e_{1}$ y $R_{2}=B+e_{2}$ y por lo tanto $\Omega=B_{M}$.

Teorema 1.3.4 Si $P$ es un conjunto de $n \geq 3$ puntos en el plano en posición general, entonces $\mathcal{T}(P)$ tiene un clan de tamaño $n$.

\section{DEMOSTRACIÓN}

Sea $n \geq 3, Q$ un polígono simple con conjuntos de vértices $P$ y $U$ su correspondiente ciclo plano en $K(P)$. 
Como $U$ es un uniciclo generador plano de $K(P)$ con exactamente un ciclo ( $U$ mismo) entonces por el Teorema 1.3.1 $\Omega=U_{U}$ es un clan de $\mathcal{T}(P)$ de tamaño $n$.

Teorema 1.3.5 Si $P$ es un conjunto de $n$ puntos en el plano en posición general con $k$ puntos interiores, entonces existe una triangulación $D$ sobre $P$ cuyas aristas son las aristas de un bosque generador plano $B$ de $K(P)$, con exactamente dos componentes $A_{1}$ y $A_{2}$, junto con un conjunto $M$ de $|P|+k-1$ aristas con un extremo en $A_{1}$ y el otro en $A_{2}$.

\section{DEMOSTRACIÓN}

Si todos los $n$ puntos de $P$ pertenecen a la frontera de la cerradura convexa de $P$, entonces definimos $B$ como el bosque generador plano de $K(P)$ con dos componentes conexas, una dada por el vértice $v_{1}$ y la otra por la trayectoria $v_{2}, v_{3}, \ldots, v_{n}$, donde $P=\left\{v_{1}, v_{2}, \ldots, v_{n}\right\}$. Definimos $M=\left\{v_{1} v_{k} \mid k=\right.$ $2, \ldots, n\}$. Es claro que las aristas de $B$ junto con las de $M$ forman una triangulación de $P$. Ver figura 1.6.

Supongamos el resultado válido, siempre que el conjunto $P^{\prime}$ tenga $t$ puntos interiores.

Sea $P=\left\{v_{1}, v_{2}, \ldots, v_{n}\right\}$ un conjunto con exactamene $t+1$ puntos interiores. Sin pérdida de generalidad, podemos suponer que $v_{n}$ es un punto interior de $P$. Claramente $P^{\prime}=\left\{v_{1}, v_{2}, \ldots, v_{n-1}\right\}$ es un conjunto con $t$ puntos interiores; por hipótesis de inducción, existe una triangulación $D^{\prime}$ sobre 


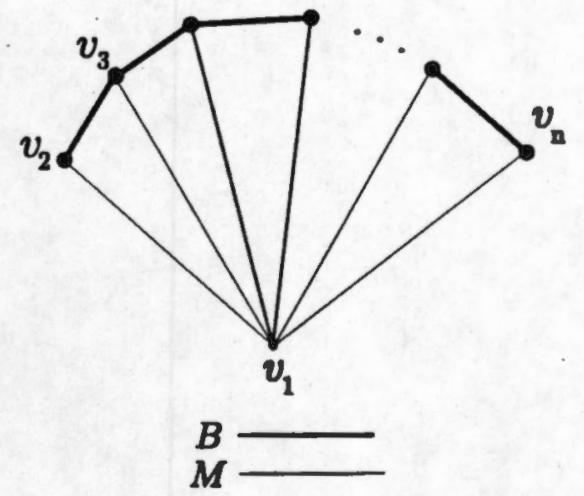

Figura 1.6

$P^{\prime}$ cuyas aristas son aristas de un bosque generador plano $B^{\prime}$ de $K\left(P^{\prime}\right)$ con exactamente dos componentes $A_{1}^{\prime}$ y $A_{2}^{\prime}$ junto con un conjunto de aristas $M^{\prime}$ de $\left|P^{\prime}\right|+t-1$ elementos, con un extremo en $A_{1}^{\prime}$ y el otro en $A_{2}^{\prime}$.

Sean $v_{i}, v_{j}, v_{k} \in P^{\prime}$ tales que $v_{n}$ está contenido en la cara triangular de $D^{\prime}$ determinada por ellos. Como $A_{1}^{\prime}$ y $A_{2}^{\prime}$ no contienen ciclos y toda arista de $M^{\prime}$ tiene un extremo en $A_{1}^{\prime}$ y el otro en $A_{2}^{\prime}$, entonces sin pérdida de generalidad, $v_{k} \in V\left(A_{1}^{\prime}\right)$ y $v_{i}, v_{j} \in V\left(A_{2}^{\prime}\right)$.

Sean $A_{1}$ la gráfica obtenida de $A_{1}^{\prime}$ al agregarle el vértice $v_{n}$ y la arista $v_{n} v_{k}, A_{2}=A_{2}^{\prime}, B=A_{1} \cup A_{2}$ y $M=M^{\prime} \cup\left\{v_{n} v_{i}, v_{n} v_{j}\right\}$.

Claramente $B$ es un bosque generador plano de $K(P)$ con exactamente dos componentes conexas $A_{1}$ y $A_{2}$. Como $v_{n} \in V\left(A_{1}\right)$ y $v_{i}, v_{j} \in V\left(A_{2}\right)$ entonces todas las aristas de $M$ tienen un extremo en $A_{1}$ y un extremo en $A_{2}$. Como $E(B) \cup M$ forman una triangulación $D$ sobre $P$. y

$$
\begin{aligned}
|M| & =\left|M^{\prime}\right|+2=\left|P^{\prime}\right|+t-1+2 \\
& =\left|P^{\prime}\right|+t+1
\end{aligned}
$$




$$
\begin{aligned}
& =|P|-1+t+1 \\
& =|P|+(t+1)-1
\end{aligned}
$$

y el resultado se sigue.

Como consecuencia inmediata del teorema anterior tenemos el siguiente corolario.

Corolario 1.3.6 Si $P$ es un conjunto de $n \geq 2$ puntos en posición general en el plano, con exactamente $k$ puntos interiores, entonces $\mathcal{T}(P)$ tiene un clan de tamaño al menos $n+k-1$.

Por el teorema 1.3.4 y el corolario 1.3.6 tenemos que si $k$ es el número de puntos interiores de $P$ entonces

$$
\omega(\mathcal{T}(P)) \geq \operatorname{máx}\{n, n+k-1\}
$$

Además si $\Omega$ es un clan de $\mathcal{T}(P)$ entonces, por teorema 1.3.3, $\Omega=U_{c}$ o $\Omega=B_{M}$. Por lo tanto $|V(\Omega)| \leq n \circ|V(\Omega)| \leq|M|$. Pero $|M| \leq\left|E\left(K_{m, \ell}\right)\right|$, donde $K_{m, \ell}$ es la gráfica bipartita completa y $n=m+\ell$. Como $\left|E\left(K_{m, \ell}\right)\right| \leq$ $\frac{n^{2}}{4}$ entonces $|M| \leq \frac{n^{2}}{4}$. Por lo tanto

$$
\omega(\mathcal{T}(P)) \leq \operatorname{máx}\left\{n, \frac{n^{2}}{4}\right\}
$$

Sea $\mathcal{T}^{*}(P)$ la subgráfica generadora de $\mathcal{T}(P)$ en la cual dos árboles son adyacentes si uno es obtenido del otro intercambiando dos aristas que se intersectan a lo más en sus extremos. 
Las mismas pruebas usadas en el caso de $\mathcal{T}(P)$ demuestran en $\mathcal{T}^{*}(P)$ que si $|P|=n$ y $k$ es el número de puntos interiores de $P$ entonces

a) $\delta\left(\mathcal{T}^{*}(P)\right) \geq 2(n+k-2)$

b) $\Delta\left(\mathcal{T}^{*}(P)\right) \leq \frac{(n-1)(n-2)(n+3)}{6}$

c) Si $U$ es un uniciclo generador plano de $K(P)$ y $C$ es el único ciclo de $U$, entonces la subgráfica $U_{C}$ de $\mathcal{T}^{*}(P)$ inducida por el conjunto de vértices $\{U-e \mid e \in C\}$ es un clan de $\mathcal{T}^{*}(P)$.

d) Si $B$ es un bosque generador plano de $K(P)$ con exactamente dos componentes conexas y $M^{*}$ es un conjunto maximal de aristas $e$ de $K(P)$ tal que $B+e$ es un árbol generador plano de $K(P)$ y tal que cualesquiera dos aristas de $M^{*}$ se intersectan a lo más en sus extremos entonces la subgráfica $B_{M^{*}}$ de $\mathcal{T}^{*}(P)$ inducida por el conjunto $\left\{B+e \mid e \in M^{*}\right\}$ es un clan de $\mathcal{T}^{*}(P)$.

e) Los teoremas 1.3 .3 y 1.3.4 siguen siendo válidos si en lugar de $\mathcal{T}(P)$ y $M$ escribimos $\mathcal{T}^{*}(P)$ y $M^{*}$ respectivamente.

f) Por último, en el teorema 1.3.5, $M$ es un conjunto maximal de aristas tal que cualesquiera dos de ellas se intersectan a lo más en sus extremos y por lo tanto $\mathcal{T}^{*}(P)$ no contiene clanes de tamaño mayor que máx $\{n, n+k-1\}$. Por lo tanto

$$
\omega\left(\mathcal{T}^{*}(P)\right)=\operatorname{máx}\{n, n+k-1\}
$$




\subsection{Conexidad de $\mathcal{T}_{B}(P)$.}

D. Avis y K. Fukuda demostraron en [2] que $\mathcal{T}(P)$ la gráfica de árboles de $P$ siempre es conexa.

Una variación de este problema es la siguiente: si $B$ es un bosque generador plano de $K(P)$ entonces definimos $\mathcal{T}_{B}(P)$ como la gráfica que tiene un vértice por cada árbol generador plano de $K(P)$ que contiene a $B$ y en la cual dos árboles $R$ y $S$ son adyacentes si $R=(S+r)-s$ con $r \in E(R) \backslash E(B)$ y $s \in E(S) \backslash E(B)$.

Demostraremos que $\mathcal{T}_{B}(P)$ también es conexa.

Si $G$ es una gráfica con conjunto de vértices $V$ y $X, X^{\prime}$ son subconjuntos de $V$, denotamos por $G\left[X, X^{\prime}\right]$, al conjunto de aristas de $G$ con un extremo en $X$ y el otro en $X^{\prime}$.

Un conjunto de corte de $G$, es un conjunto de la forma $G\left[X, X^{\prime}\right]$, donde $X$ es un subconjunto propio no vacío de $V$ y $X^{\prime}=V-X$.

En particular si $K(P)$ es la gráfica geométrica completa sobre $P$ y $D$ es una triangulación sobre $P$ entonces $K\left[X, X^{\prime}\right]$ y $D\left[X, X^{\prime}\right]$ denotarán al conjunto de corte de $K(P)$ y de $D$ respectivamente.

Lema 1.4.1 Si $D$ es una triangulación sobre $P$ y $K\left[X, X^{\prime}\right]$ es un conjunto de corte de $K(P)$, entonces para cualquier arista e en $K\left[X, X^{\prime}\right]$, existe una arista $e^{\prime}$ en $D\left[X, X^{\prime}\right]$ tal que e y $e^{\prime}$ no se intersectan, excepto posiblemente en sus extremos. 
Sea $e=u v$ una arista en $K\left[X, X^{\prime}\right]$, con $u \in X$ y $v \in X^{\prime}$. Definimos $e^{*}=y z$ como la primera arista en $D\left[X, X^{\prime}\right]$ que intersecta a $e$ en puntos distintos a sus extremos y que encontramos al recorrer $e$ de $u$ hacia $v$. Sin pérdida de generalidad $y \in X$ y $z \in X^{\prime}$. Sean $L$ la recta que pasa por yz y $L_{1}$ el semiplano cerrado determinado por $L$ y en el que se encuentra $u$. Como la arista $y z$ es intersectada por $u v$ entonces yz no está en la frontera de $C H(P)$, por lo tanto $y z$ es lado de dos caras triangulares de $D$. Sea $C=x y z$ la cara triangular de $D$, que tiene a $y z$ como uno de sus lados y que se encuentra en $L_{1}$. Ver figura 1.7. Como $e^{*}$ es la primera arista de $D\left[X, X^{\prime}\right]$ que $e$ intersecta tenemos que: si $x \in X$ entonces $e^{\prime}=x z \in D\left[X, X^{\prime}\right]$ no intersecta a $e$ ó si $x \in X^{\prime}$ enontces $e^{\prime}=x y \in D\left[X, X^{\prime}\right]$ no intersecta a $e$. Por lo tanto el lema se sigue.

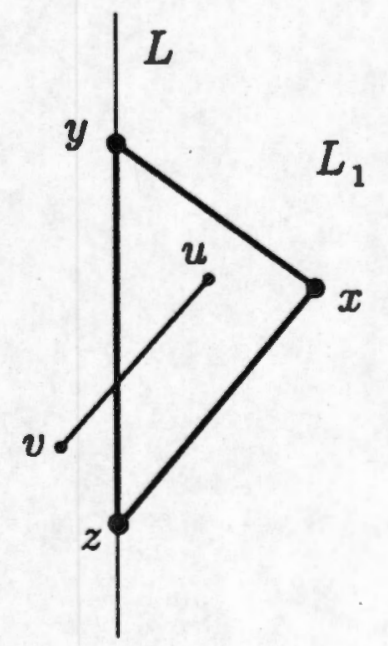

Figura 1.7

Denotaremos por $\mathcal{T}_{B}$ al conjunto de vértices de $\mathcal{T}_{B}(P)$. Si $K\left[X, X^{\prime}\right]$ es 
un conjunto de corte de $K(P)$ tal que ninguna de sus aristas pertenece a $B$ y $e$ es una arista de $K\left[X, X^{\prime}\right]$, definimos $\mathcal{T}_{e}$ como la colección de árboles generadores planos en $\mathcal{T}_{B}$ que contienen a $e$ como una de sus aristas.

Claramente $\mathcal{T}_{e} \subseteq \mathcal{T}_{B}$. Además si $R$ es un árbol generador plano en $\mathcal{T}_{B}$, existe una arista $e^{*}$ de $R$ tal que $e^{*} \in K\left[X, X^{\prime}\right]$ y por lo tanto $R \in \mathcal{T}_{\mathrm{e}^{*}}$. De aquí tenemos que $\mathcal{T}_{B}=\bigcup_{e \in K\left[X, X^{\prime}\right]} \mathcal{T}_{e}$.

Teorema 1.4.2 $\mathcal{T}_{B}(P)$ es conexa.

\section{DEMOSTRACIÓN}

Usaremos inducción sobre $\omega(B)$, el número de componentes de $B$.

Si $\omega(B)=1$, entonces $B$ es un árbol generador plano de $P$ y por lo tanto $\mathcal{T}_{B}(P)$ tiene a $B$ como único vértice.

Supongamos el resultado válido siempre que $\omega(B)=m-1$. Sean $B$ un bosque generador plano de $P$ con exactamente $m$ componentes y $B_{1}$ una componente conexa de $B$. Definimos $X$ como el conjunto de vértice de $B_{1} \mathrm{y}$ $X^{\prime}=P \backslash X$. Claramente $K\left[X, X^{\prime}\right]$ es un conjunto de corte de $K(P)$ que no contiene aristas de $B$, por lo tanto $\mathcal{T}_{B}=\bigcup_{e \in K\left[X, X^{\prime}\right]} \mathcal{T}_{e}$.

Para cualquier arista $e$ en $K\left[X, X^{\prime}\right], \omega(B+e)=m-1$ por lo tanto por hipótesis de inducción, para cualquier arista $e$ en $K\left[X, X^{\prime}\right], \mathcal{T}_{B+e}(P)$ es conexa. Por definición el conjunto de vértices de $\mathcal{T}_{B+e}(P)$ es $\mathcal{T}_{e}$ para cada $e \in K\left[X, X^{\prime}\right]$. 
Demostraremos que si $e_{1}, e_{2}$ son dos aristas de $K\left[X, X^{\prime}\right]$ entonces para cualesquiera dos vértices en $\mathcal{T}_{B+e_{1}} \cup \mathcal{T}_{B+e_{2}}$ existe una trayectoria en $\mathcal{T}_{B}(P)$ que los une.

Sean $e_{1}, e_{2}$ dos aristas en $K\left[X, X^{\prime}\right]$. Podemos suponer que ambas aristas no intersectan a $B$, de otra forma no hay nada que demostrar.

CASO 1. Si $e_{1}, e_{2}$ son dos aristas en $K\left[X, X^{\prime}\right]$ que no se intersectan en puntos distintos de sus extremos, entonces $B+e_{1}$ no contiene ciclos y por lo tanto, $B+e_{1}+e_{2}$ es acíclico o contiene un único ciclo plano. Ver figura 1.8.
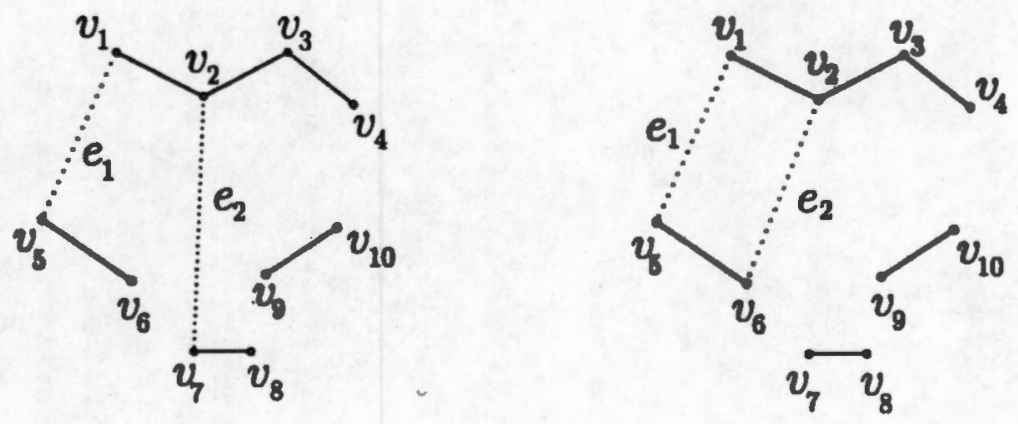

$$
\begin{aligned}
& X=\left\{v_{1}, v_{2}, v_{3}, v_{4}\right\} \\
& X^{\prime}=\left\{v_{5}, \ldots, v_{10}\right\}
\end{aligned}
$$

$e_{1}, e_{2}$ no se intersectan

Figura 1.8

CASO 1a. Si $B+e_{1}+e_{2}$ es acíclico, entonces cualquier árbol generador $T$ que contiene a $B+e_{1}+e_{2}$ es un vértice de $\mathcal{T}_{B+e_{1}}(P)$ y de $\mathcal{T}_{B+e_{2}}(P)$ y el teorema se sigue.

CASO 1b. Si $B+e_{1}+e_{2}$ contiene un único ciclo $C$, entonces $e_{1}$ y $e_{2}$ pertenecen a $C$. Sea $D$ una triangulación de $B+e_{1}+e_{2}$ y $T$ un árbol 
generador de $D$ que contiene a $B+e_{1}$. Por lo tanto $T$ no tiene a $e_{2}$ como arista y $T+e_{2}$ contiene a $B+e_{1}+e_{2}$, por lo tanto $T+e_{2}$ contiene a $C$ y este es el único ciclo de $T+e_{2}$. Sea $T^{\prime}=T+e_{2}-e_{1}$, por lo tanto $T^{\prime}$ está en $\mathcal{T}_{B+e_{2}}(P)$. Como $\mathcal{T}_{B+e_{1}}(P)$ y $\mathcal{T}_{B+e_{2}}(P)$ son conexas y $T, T^{\prime}$ son adyacentes en $\mathcal{T}_{B}(P)$ entonces para cualesquiera dos vértices de $\mathcal{T}_{B+e_{1}} \cup \mathcal{T}_{B+\text { eq }_{2}}$ existe una trayectoria que los une en $\mathcal{T}_{B}(P)$.

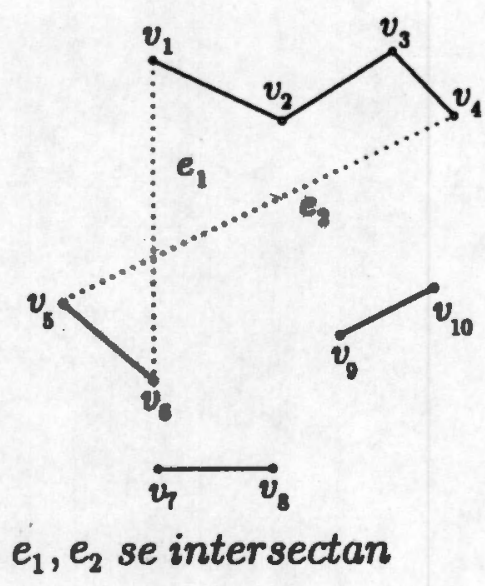

a)

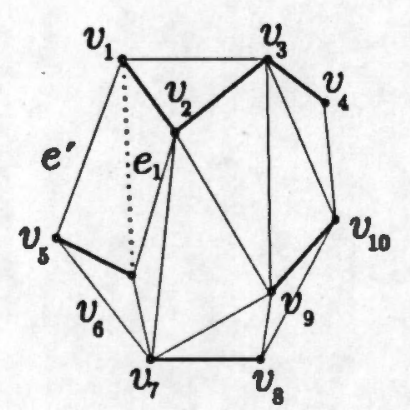

$D$ una triangulación de $B+e_{1}$

b)

Figura 1.9

CASO 2. Si $e_{1}, e_{2}$ son dos aristas de $K\left[X, X^{\prime}\right]$ que se intersectan en puntos distintos de sus extremos, ver figura 1.9a., entonces sea $D$ una triangulación cualquiera de $B+e_{1}$ y $D\left[X, X^{\prime}\right]$ el corte definido por $X$ y $X^{\prime}$ en $D$. Como $e_{2}$ es una arista de $K\left[X, X^{\prime}\right]$, por lema 1.4 .1 , existe $e^{\prime} \in D\left[X, X^{\prime}\right]$ tal que $e_{2}$ y $e^{\prime}$ no se intersectan en puntos distintos de sus extremos, ver figura 1.9b. Además como $e_{1}$ y $e^{\prime}$ son aristas de $D$ tampoco se intersectan en puntos distintos de sus extremos, por lo tanto como en el caso 1, para cualesquicra dos vértices en $\mathcal{T}_{B+e_{1}} \cup \mathcal{T}_{B+e^{\prime}}$ y en $\mathcal{T}_{B+e^{\prime}} \cup \mathcal{T}_{B+e_{2}}$ existe una trayectoria que los 
une en $\mathcal{T}_{B}(P)$ y por lo tanto para cualesquiera dos vértices en $\mathcal{T}_{B+e_{1}} \cup \mathcal{T}_{B+e_{2}}$ existe una trayectoria que los une en $\mathcal{T}_{B}(P)$. 


\section{Capítulo 2.}

\section{Ciclos Hamiltonianos en la gráfica de tra- yectorias.}

\subsection{Introducción}

R.L. Cummings en [5] demostró que para la gráfica de árboles $T(G)$, de una gráfica abstracta conexa $G$, existe un ciclo que pasa por todos los vértices de $T(G)$ es decir, $T(G)$ es hamiltoniana.

En el caso de gráficas geométricas C. Hernando et al en [7] probaron que si $P$ es una colección de puntos en posición convexa, la gráfica de árboles $\mathcal{T}(P)$ de $P$ es hamiltoniana.

Si $P$ es un conjunto de $n$ puntos en posición conexa y $T(P)$ es la subgráfica de $\mathcal{T}(P)$, inducida por el conjunto de trayectorias generadoras planas de $K(P)$, entonces demostraremos que $T(P)$ también es hamiltoniana. A $T(P)$ la llamaremos la gráfica de trayectorias de $P$.

Como para cualquier trayectoria $S$ de $P$, la planaridad depende sólo de la posición relativa de los vértices de $P$, a lo largo de la frontera de la cerradura convexa de $P$, entonces para cualquier conjunto $P$ de $n$ puntos en posición convexa en el plano, la gráfica $T(P)$ es isomorfa a $T\left(P_{n}\right)$, donde $P_{n}$ es un polígono regular con $n$ puntos. Demostraremos entonces que $T\left(P_{n}\right), n \geq 3$, contiene un ciclo hamiltoniano. Las gráficas $T\left(P_{3}\right)$ y $T\left(P_{4}\right)$ se muestran en la figura 2.1. 

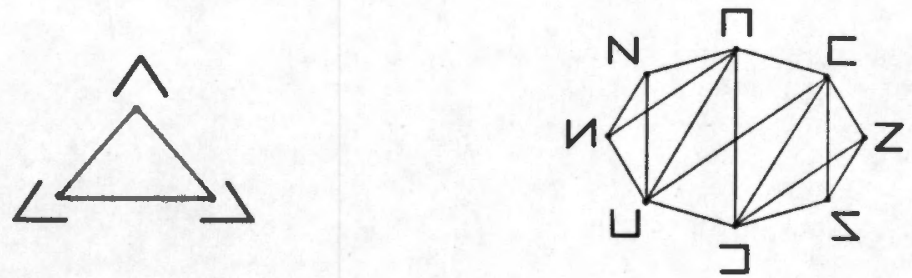

Las gráficas $T\left(P_{3}\right)$ y $T\left(P_{4}\right)$

Figura 2.1

\subsection{Ciclos Hamiltonianos.}

Para poder establecer que $T\left(P_{n}\right)$ es una gráfica hamiltoniana, demostraremos algunos resultados previos,

Si $w_{1}, w_{2}, \ldots, w_{n}$ son los vértices de $P_{n}$, ordenados en el sentido en que se mueven las manecillas del reloj y donde los subíndices son sumados módulo $n$, entonces para cualesquiera dos vértices $w_{t}$ y $w_{s}$ de $P_{n}$, denotamos por $\left[w_{t}, w_{s}\right]$ al conjunto $\left\{w_{t}, w_{t+1}, w_{t+2}, \ldots, w_{s}\right\}$.

En [7], C. Hernando et al, probaron el siguiente resultado.

Teorema 2.2.1 Sea $P$ un conjunto de $n$ puntos en el plano en posición convexa, $n \geq 3$. Si $R$ es un árbol generador plano de $K(P)$, entonces $R$ tiene al menos dos aristas terminales en la frontera de $C H(P)$.

Como consecuencia de este teorema tenemos el siguiente corolario. 
Corolario 2.2.2 Sea $P$ un conjunto de $n$ puntos en el plano en posición convexa. Si e es una arista terminal de una trayectoria generadora plana de $T$ de $K(P)$, entonces e está en la frontera de $C H(P)$.

\section{DEMOSTRACIÓN}

Si $n \leq 3$, entonces todas las aristas están en la frontera de $C H(P)$. Si $n \geq 3$, entonces $T$ tiene exactamente dos aristas terminales las cuales por el teorema 2.2.1, están en la frontera dè $C H(P)$.

Sea $T=w_{1}, w_{t_{2}}, \ldots, w_{t_{n}}$ una trayectoria generadora plana de $K\left(P_{n}\right)$ con un extremo en $w_{1}$. Como $w_{1}, w_{t_{2}}$ es una arista terminal de $T$, entonces por el corolario 2.2.2 $w_{1} w_{t_{2}}$ está en la frontera de $C H\left(P_{n}\right)$. Sin pérdida de generalidad, $w_{t_{2}}=w_{2}$.

Si $w_{3} \neq w_{t_{3}} \neq w_{n}$, entonces $w_{3}$ y $w_{n}$ están separados por la línea $\ell$ que contiene la arista $w_{2} w_{t_{3}}$. Como $T$ es una trayectoria generadora plana de $P_{n}$, entonces $w_{3}$ está conectada a $w_{t_{3}}$ por una subtrayectoria de $T$ contenida en uno de los semiplanos definidos por $\ell$, y $w_{n}$ está conectado a $w_{t_{3}}$ por una subtrayectoria de $T$ contenida en el otro semiplano definido por $\ell$ lo cual no es posible, ya que todos los vértices de $T$ son de grado a lo más dos. Por lo tanto $w_{t_{3}}=w_{3} \circ w_{t_{3}}=w_{n}$, es decir, los primeros tres vértices de $t$ son vértices consecutivos de $P_{n}$. Siguiendo este razonamiento tenemos el siguiente teorema.

Teorema 2.2.3 Si $T$ es una trayectoria generadora plana de $K\left(P_{n}\right)$ entonces para toda $k=1,2, \ldots, n$ los primeros $k$ vértices de $T$ son puntos consecutivos de $P_{n}$. 
Para cualquier trayectoria generadora plana $T=w_{t_{1}}, w_{t_{2}}, \ldots, w_{t_{n}}$ de $K\left(P_{n}\right)$ y para cualquier entero $1 \leq k \leq n$, denotamos por $T_{k}$ a la subtrayectoria $w_{t_{1}}, w_{t_{2}}, \ldots, w_{t_{k}}$ de $T$ y por $T^{k}$ a la subtrayectoria $w_{t_{k}}, w_{t_{k+1}}, \ldots, w_{t_{n}}$ de $T$.

Sea $X=\left\{x_{1}, x_{2}, \ldots, x_{s}\right\}$ un conjunto de puntos en el plano en posición convexa y sea $\theta: X \longrightarrow X$ la función dada por $\theta\left(x_{k}\right)=x_{s+1-k}$. Para cualquier gráfica geométrica $G$ con conjunto de vértices $X$, denotamos por $\theta(G)$ a la gráfica geométrica, con conjunto de vértices $X$ y en la cual $\theta\left(x_{i}\right)$ y $\theta\left(x_{j}\right)$ son adyacentes en $G$ si y sólo si $x_{i}$ y $x_{j}$ son adyacentes en $G$. En el caso en que $X$ es un conjunto de puntos consecutivos de $P_{n}, \theta(G)$ es la reflexión de $G$ con respecto a la recta ortogonal al segmento $x_{1} x_{s}$, que bisecta a $P_{n}$. Ver ejemplo en la figura 2.2.
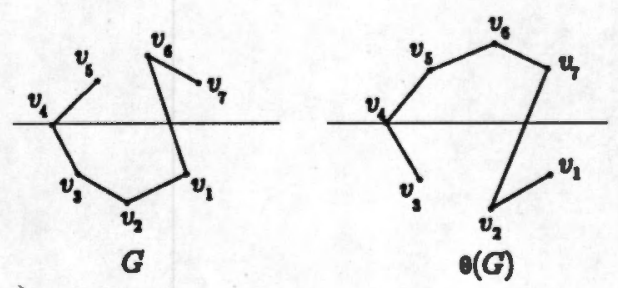

Figura 2.2

Lema 2.2.4 Sea $G$ una gráfica geométrica con conjunto de vértices $X$. Si $G$ es una gráfica plana entonces $\theta(G)$ también es plana.

\section{DEMOSTRACIÓN}

Sean $x_{i} x_{j}$ y $x_{h} x_{k}$ dos aristas distintas de $G$. Sin pérdida de generalidad, podemos suponer que $i=1<h<k$. Como $G$ es una gráfica plana entonces 
$i=1<h<k \leq j \circ i=1<j \leq h<k$. Por lo tanto $s+1-j \leq s+1-k<$ $s+1-h<s+1-i$ ó $s+1-k<s+1-h \leq s+1-j \leq s+1-i$. Esto implica que $\theta\left(x_{i}\right) \theta\left(x_{j}\right)$ y $\theta\left(x_{h}\right) \theta\left(x_{k}\right)$ no se intersectan en $\theta(G)$.

Lema 2.2.5 Sea $X$ un conjunto de puntos en el plano en posición convexa $y$ $G$ y $F$ dos gráficas geométricas sobre $X$. Si $G$ y $F$ son adyacentes entonces $\theta(G)$ y $\theta(F)$ son adyacentes.

\section{Demostración}

Si $F=\left(G-x_{i} x_{j}\right)+x_{h} x_{k}$ entonces

$$
\theta(F)=\left(\theta(G)-\theta\left(x_{i}\right) \theta\left(x_{j}\right)\right)+\theta\left(x_{h}\right) \theta\left(x_{k}\right)
$$

Si $P$ es un conjunto con $n$ puntos y $n=2 m+1$, entonces para $i=$ $1,2, \ldots, m+1$ definimos $u_{i}=w_{i}$ y $v_{i}=w_{2 m-i+2}$. Denotamos por $A_{m}$ al conjunto de trayectorias generadoras planas $T$ de $T\left(P_{2 m+1}\right)$ con punto medio $u_{m+1}=w_{m+1}=v_{m+1}$. Por teorema 2.2.3 podemos suponer sin pérdida de generalidad que $V\left(T_{m+1}\right)=\left\{u_{1}, u_{2}, \ldots, u_{m+1}\right\}$ y $V\left(T^{m+1}\right)=$ $\left\{v_{1}, v_{2}, \ldots, v_{m+1}\right\}$, para cualquier $T \in A_{m}$. A la subtrayectoria $T_{m+1}$ de $T$ la llamaremos subtrayectoria izquierda de $T$ y la denotaremos por $T_{I}$ y de manera análoga $T^{m+1}$ será llamada subtrayectoria derecha de $T$ y la denotaremos por $T_{D}$. Ver figura 2.3. 


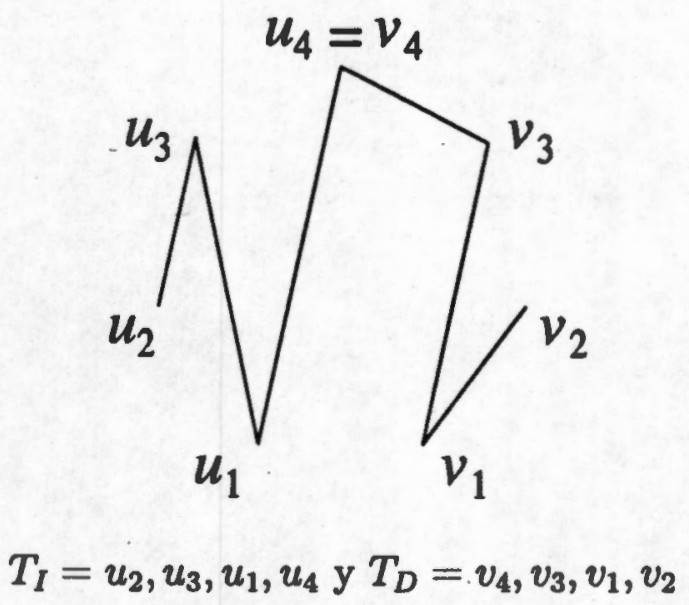

Figura 2.3

Lema 2.2.6 Dos trayectorias $T$ y $S$ en $A_{m}$ son adyacentes en $T\left(P_{2 m+1}\right)$ si y sólo si $T_{D}$ y $S_{D}$ son adyacentes y $T_{I}=S_{I}$ o bien $T_{I}$ y $S_{I}$ son adyacentes $y$ $T_{D}=S_{D}$

\section{DEMOSTRACIÓN}

Se sigue de manera inmediata del teorema 2.2.3 y de la definición de adyacencia en $T\left(P_{2 m+1}\right)$.

Demostraremos que si $\mathcal{A}_{m}$ es la subgráfica de $T\left(P_{2 m+1}\right)$ inducida por $A_{m}$ entonces $\mathcal{A}_{m}$ contiene una trayectoria hamiltoniana $J_{m}$. Para ésto daremos primero las siguientes definiciones y el siguiente lema.

Sean $I$ una trayectoria generadora plana con conjunto de vértices $\left\{u_{1}, u_{2}, \ldots, u_{m+1}\right\}$ y con $u_{m+1}$ como uno de sus extremos y $D$ una trayectoria generadora plana con conjunto de vértices $\left\{v_{1}, v_{2}, \ldots, v_{m+1}\right\}$ y con $v_{m+1}$ como uno de sus extremos. Denotaremos por $I * D$ a la trayectoria en $A_{m}$ con $I$ como subtrayectoria izquierda y con $D$ como subtrayectoria derecha. 
Para $i, j \in\{1, m\}$, definimos $A_{m}^{i, j}$ como conjunto de trayectorias en $A_{m}$ que tienen a $u_{i} u_{m+1}$ y a $v_{m+1} v_{j}$ como dos de sus aristas. Denotaremos por $\mathcal{A}_{m}^{i, j}$ a la subgráfica de $\mathcal{A}_{m}$ inducida por $A_{m}^{i, j}$.

Lema 2.2.7 Para $m \geq 2$ y $i, j \in\{1, m\}$, la gráfica $\mathcal{A}_{m}^{i, j}$ es isomorfa a $\mathcal{A}_{m-1}$.

\section{Demostración}

Para $i, j \in\{1, m\}$, definimos $\alpha_{i, j}: A_{m}^{i, j} \longrightarrow A_{m-1}$ como la función dada por $\alpha_{i, j}(T)=\theta^{\frac{m-i}{m-1}}\left(T_{I}-u_{m+1}\right) * \theta^{\frac{m-j}{m-1}}\left(T_{D}-v_{m+1}\right)$, donde $\theta^{1}=\theta$ y $\theta^{0}$ es la función identidad. Ver figura 2.4.

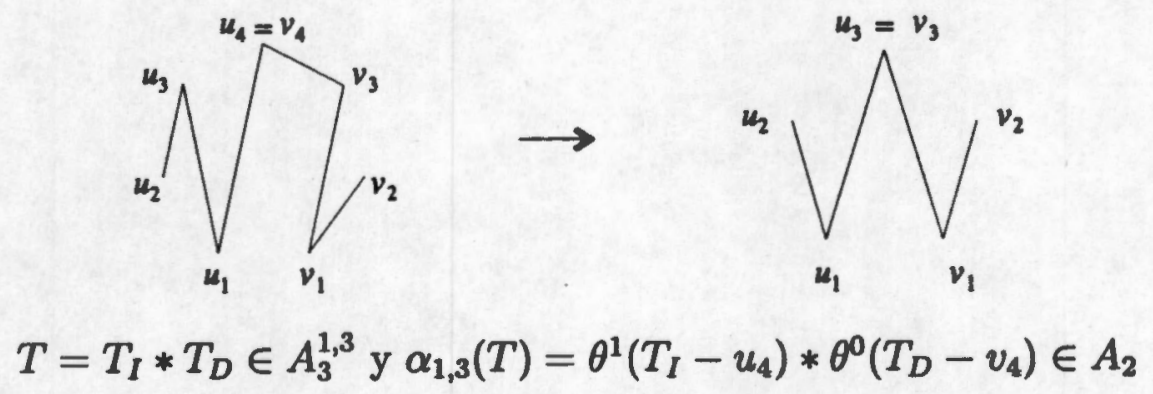

Figura 2.4

Como $u_{i} u_{m+1}$ es una arista de $T_{I}$, entonces $u_{i}$ es un vértice terminal de $T_{I}-u_{m+1}$ y como $u_{m}=\theta^{\frac{m-i}{m-1}}\left(u_{i}\right)$, entonces $u_{m}$ es un vértice terminal de $\theta^{\frac{m-i}{m-1}}\left(T_{I}-u_{m+1}\right)$. Como $v_{m+1} v_{j}$ es una arista de $T$, entonces $v_{j}$ es un vértice terminal de $T_{D}-v_{m+1}$ y como $v_{m}=\theta^{\frac{m-1}{m-1}}\left(v_{j}\right)$ entonces $v_{m}$ es un vértice terminal de $\theta^{\frac{m-j}{m-1}}\left(T_{D}-v_{m+1}\right)$. Por lo tanto $\alpha_{i, j}(T)$ es una trayectoria generadora plana de $P_{2 m-1}$ con punto medio $u_{m}=w_{m}=v_{m}$. 
Si $\alpha_{i, j}(T)=\alpha_{i, j}(S)$ entonces

$$
\begin{aligned}
& \theta^{\frac{m-i}{m-1}}\left(T_{I}-u_{m+1}\right)=\theta^{\frac{m-1}{m-1}}\left(S_{I}-u_{m+1}\right) \mathrm{y} \\
& \theta^{\frac{m-j}{m-1}}\left(T_{D}-v_{m+1}\right)=\theta^{\frac{m-1}{m-1}}\left(S_{D}-v_{m+1}\right) .
\end{aligned}
$$

Como $\theta$ y $\theta^{0}$ son inyectivas, por lo tanto $T_{I}-u_{m+1}=S_{I}-v_{m+1}$ y $T_{D}-v_{m+1}=S_{D}-v_{m+1}$, esto implica que $T_{I}=S_{I}$ y $T_{D}=S_{D}$ y por lo tanto $\alpha_{i, j}$ es inyectiva. Por otro lado, si $T^{y}=T_{I}^{\gamma} * T_{D}^{\prime} \in A_{m-1}$, definimos $T_{I}=\theta^{\frac{m-j}{m-1}}\left(T_{I}^{\prime}\right)+u_{i} u_{m+1}$ y $T_{D}=\theta^{\frac{m-j}{m-1}}\left(T_{D}^{\prime}\right)+v_{j} v_{m+1}$. Como $u_{m}$ es un vértice terminal de $T_{I}^{\prime}$ y $\theta^{\frac{m-i}{m-1}}\left(u_{m}\right)=u_{i}$ entonces $u_{i}$ es un vértice terminal de $\theta^{\frac{m-i}{m-1}}\left(T_{I}^{\prime}\right)$ y $u_{m+1}$ es vértice terminal de $T_{I}$. Como $v_{m}$ es vértice terminal de $T_{D}^{\prime} \mathrm{y}$ $\theta^{\frac{m-j}{m-1}}\left(v_{m}\right)=v_{j}$ entonces $v_{j}$ es un vértice terminal de $\theta^{\frac{m-j}{m-1}}\left(T_{D}^{\prime}\right)$ y $v_{m+1}$ es vértice terminal de $T_{D}$. Por lo tanto $T=T_{I} * T_{D}$ es una trayectoria generadora plana de $P_{2 m+1}$ con punto medio $u_{m+1}=v_{m+1}=w_{m+1}$. Como $\theta^{2}=\theta^{0}$, es la función identidad, entonces $\alpha_{i, j}(T)=T^{\prime}$ y por lo tanto $\alpha_{i, j}$ es una función suprayectiva de $A_{m}^{i, j}$ sobre $A_{m-1}$. Por lemas 2.2 .5 y 2.2.6 $T$ y $S$ son adyacentes en $\mathcal{A}_{m}^{i, j}$ si y sólo si $\alpha_{i, j}(T)$ y $\alpha_{i, j}(S)$ son adyacentes en $\mathcal{A}_{m-1}$, por lo tanto $\mathcal{A}_{m}^{i, j}$ y $\mathcal{A}_{m-1}$ son isomorfas.

Proposición 2.2.8 Si $m \geq 2$, entonces $\mathcal{A}_{m}$ contiene una trayectoria hamiltoniana $J_{m}$ con extremos $I_{m} * D_{m}$ y $I_{m} * D_{m}^{\prime}$, donde $I_{m}=u_{1}, u_{2}, \ldots, u_{m+1}$, $D_{m}=v_{m+1}, v_{m}, \ldots, v_{1}$ y $D_{m}^{\prime}=v_{m+1}, v_{1} v_{2}, \ldots, v_{m}$.

\section{DEMOSTRACIÓN}

Para $k=2, \mathcal{A}_{2}$ es mostrada en la figura 2.5 . 


\section{$\bigcap-\downarrow-w-\Uparrow$}

La trayectoria $J_{2}$

Figura 2.5

Supongamos que el resultado es válido para $\mathcal{A}_{m-1}$ con $m \geq 3$.

Por inducción $\mathcal{A}_{m-1}$ contiene una trayectoria hamiltoniana $J_{m-1}$ con extremos en $I_{m-1} * D_{m-1}$ y $I_{m-1} * D_{m-1}^{\prime}$ por lo tanto $\mathcal{A}_{m}^{i, j}$ contiene una trayectoria hamiltoniana $J_{m}^{i, j}$ con extremos $\alpha_{i, j}^{-1}\left(I_{m-1} * D_{m-1}\right)$ y $\alpha_{i, j}^{-1}\left(I_{m-1} * D_{m-1}^{\prime}\right)$. Para terminar la prueba mostraremos como concatenar las trayectorias $J_{m}^{m, m}, J_{m}^{1, m}$, $J_{m}^{1,1}, J_{m}^{m, 1}$ para formar una trayectoria hamiltoniana $J_{m}$ de $\mathcal{A}_{m}$ con extremos

$$
\begin{aligned}
& I_{m} * D_{m}=\alpha_{m, m}^{-1}\left(I_{m-1} * D_{m-1}\right) \text { y } \\
& I_{m} * D_{m}^{\prime}=\alpha_{m, 1}^{-1}\left(I_{m-1} * D_{m-1}\right)
\end{aligned}
$$

Como

$$
\begin{aligned}
\theta^{-1}\left(I_{m-1}\right) & =\theta\left(I_{m-1}\right)=I_{m-1} \mathrm{y} \\
\theta^{-1}\left(D_{m-1}\right) & =\theta\left(D_{m-1}\right)=D_{m-1}
\end{aligned}
$$

entonces $\alpha_{m, m}^{-1}\left(I_{m-1} * D_{m-1}^{\prime}\right)=\left(I_{m-1}+u_{m} u_{m+1}\right) *\left(D_{m-1}^{\prime}+v_{m} v_{m+1}\right)$ es adyacente a $\left(I_{m-1}+u_{1} u_{m+1}\right) *\left(D_{m-1}^{\prime}+v_{m} v_{m+1}\right)=\alpha_{1, m}^{-1}\left(I_{m-1} * D_{m-1}^{\prime}\right)$. De manera análoga $\alpha_{1, m}^{-1}\left(I_{m-1} * D_{m-1}\right)$ es adyacente a $\alpha_{1,1}^{-1}\left(I_{m-1} * D_{m-1}\right)$ y también $\alpha_{1,1}^{-1}\left(I_{m-1} * D_{m-1}^{\prime}\right)$ es adyacente a $\alpha_{m, 1}^{-1}\left(I_{m-1} * D_{m-1}^{\prime}\right)$.

Si $P$ es un conjunto con $n$ puntos y $n=2 m$ entonces para $i=1,2, \ldots, m$ definimos $u_{i}=w_{i}$ y $v_{i}=w_{2 m-i+1}$. Para $i, j \in\{1, m\}$, definimos como $B_{m}^{i, j}$ al 
conjunto de trayectorias generadoras planas $T$ de $T\left(P_{2 m}\right)$ que tienen a $u_{i} v_{j}$ como arista media y tal que $V\left(T_{m}\right)=\left\{u_{1}, \ldots, u_{m}\right\}$ con $u_{i}$ como uno de los vértices terminales de $T_{m}$ y $V\left(T^{m+1}\right)=\left\{v_{1}, v_{2}, \ldots, v_{m}\right\}$, con $v_{j}$ como uno de los vértices terminales de $T^{m+1}$.

En este caso a $T_{m}$ la llamaremos la subtrayectoria izquierda y a $T^{m+1}$ la subtrayectoria derecha y las denotaremos por $T_{I}$ y $T_{D}$ respectivamente.

Lema 2.2.9 Dos trayectorias $S, T$ en $B_{m}^{i, j}$ son adyacentes en $T\left(P_{2 m}\right)$ si $y$ sólo si $S_{D}$ y $T_{D}$ son adyacentes y $S_{I}=T_{I}$ o bien $S_{I}$ y $T_{I}$ son adyacentes $y$ $S_{D}=T_{D}$

\section{DEMOSTRACIÓN}

Análoga a la del lema 2.2.6.

Sean $I$ una trayectoria generadora plana con conjunto de vértices $\left\{u_{1}, u_{2}, \ldots, u_{m}\right\}$ y con $u_{i}$ como uno de sus vértices terminales (con $i \in\{1, m\}$ ) y $D$ una trayectoria generadora plana con conjunto de vértices $\left\{v_{1}, v_{2}, \ldots, v_{m}\right\}$ y con $v_{j}$ como uno de sus extremos (con $j \in\{1, m\}$ ). Denotaremos por $I * u_{i} v_{j} * D$ a la trayectoria en $B_{m}^{i, j}$ que tiene a $I$ como subtrayectoria izquierda y a $D$ como subtrayectoria derecha.

Lema 2.2.10 Para $m \geq 3, i, j \in\{1, m\}$, la subgráfica $\mathcal{B}_{m}^{i, j}$ de $T\left(P_{2 m}\right)$ inducida por $B_{m}^{i, j}$ es isomorfa a $\mathcal{A}_{m-1}$. 


\section{DEMOSTRACIÓN}

Para $i, j \in\{1, m\}$, definimos $\beta_{i, j}: B_{m}^{i, j} \rightarrow A_{m-1}$ como la función dada por $\beta_{i, j}(T)=\theta^{\frac{m-i}{m-1}}\left(T_{I}\right) * \theta^{\frac{m-j}{m-1}}\left(T_{D}\right)$.

Como $u_{i}$ es un vértice terminal de $T_{I}$ y $\theta^{\frac{m-i}{m-1}}\left(u_{i}\right)=u_{m}$, entonces $u_{m}$ es un vértice terminal de $\theta^{\frac{m-i}{m-1}}\left(T_{I}\right)$ y como $v_{j}$ es un vértice terminal de $T_{D}$ y $v_{m}=\theta^{\frac{m-j}{m-1}}\left(v_{j}\right)$, entonces $v_{m}$ es un vértice terminal de $\theta^{\frac{m-j}{m-1}}\left(T_{D}\right)$. Por lo tanto el punto medio de $\beta_{i, j}(T)$, es $u_{m}=w_{m}=v_{m}$, lo cual implica que $\beta_{i, j}(T) \in A_{m-1}$.

Si $\beta_{i, j}(S)=\beta_{i, j}(T)$, entonces $\theta^{\frac{m-i}{m-1}}\left(S_{I}\right)=\theta^{\frac{m-i}{m-1}}\left(T_{I}\right)$ y $\theta^{\frac{m-j}{m-1}}\left(S_{D}\right)=\theta^{\frac{m-j}{m-1}}\left(T_{D}\right)$. Como $\theta$ y $\theta^{0}$ son funciones inyectivas entonces $S_{I}=T_{I}$ y $S_{D}=T_{D}$ y por lo tanto $\beta_{i, j}$ es inyectiva.

Sea $T^{\prime}=T_{I}^{\prime} * T_{D}^{\prime} \in A_{m-1}$. Como $u_{m}=v_{m}$ es el punto medio de $T^{v}$ entonces $u_{m}$ es vértice terminal de $T_{I}^{\prime}$ y $v_{m}$ es vértice terminal de $T_{D}^{\prime}$; por lo tanto como $\theta^{\frac{m-i}{m-1}}\left(u_{m}\right)=u_{i}$, $u_{i}$ es vértice terminal de $\theta^{\frac{m-i}{m-1}}\left(T_{I}^{\prime}\right)$ y como $\theta^{\frac{m-j}{m-1}}\left(v_{m}\right)=v_{j}, v_{j}$ es un vértice terminal de $\theta^{\frac{m-j}{m-1}}\left(T_{D}^{\prime}\right)$. Esto implica que $T=\theta^{\frac{m-i}{m-1}}\left(T_{I}^{\prime}\right) * u_{i} v_{j} * \theta^{\frac{m-j}{m-1}}\left(T_{. D}^{\prime}\right)$ es un elemento de $B_{m}^{i, j}$. Como $\theta^{2}=\theta^{0}$ es la función identidad entonces $\beta_{i, j}(T)=T^{\prime}$ y por lo tanto $\beta_{i, j}$ es una función suprayectiva.

Por lemas 2.2.5 y 2.2.9, $S$ y $T$ son adyacentes en $\mathcal{B}_{m}^{i, j}$ si y sólo si $\beta_{i, j}(T)$ y $\beta_{i, j}(S)$ son adyacentes en $\mathcal{A}_{m-1}$. Por lo tanto $\mathcal{B}_{m}^{i, j}$ y $\mathcal{A}_{m-1}$ son isomorfas.

Sea $B_{m}=B_{m}^{m, m} \cup B_{m}^{1, m} \cup B_{m}^{m, 1} \cup B_{m}^{1,1}$ y $\mathcal{B}_{m}$ la subgráfica de $T\left(P_{2 m}\right)$ inducida por $B_{m}$. Ver figura 2.6 . 


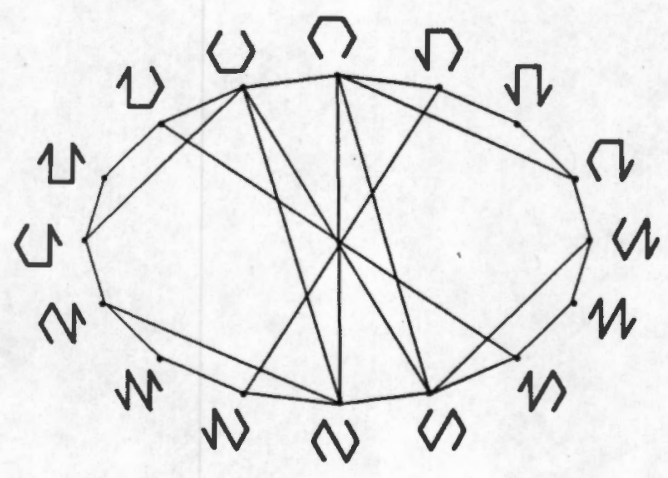

La gráfica $\mathcal{B}_{m}$

Figura 2.6

Sea $C$ el ciclo $u_{1}, u_{2}, \ldots, u_{m}, v_{m}, v_{m-1}, \ldots, v_{1}$ y $S_{m}$ y $S_{m}^{\prime}$ las trayectorias $C-u_{1} v_{1}$ y $C-u_{m} v_{m}$ respectivamente.

Demostraremos ahora que $\mathcal{B}_{m}$ contiene una trayectoria hamiltoniana.

Proposición 2.2.11 Para $m \geq 3$, la gráfica $\mathcal{B}_{m}$ contiene una trayectoria hamiltoniana $K_{m}$ con extremos $S_{m}$ y $S_{m}^{\prime}$.

\section{DEMOSTRACIÓN}

Por lema 2.2.10 tenemos que cada $\mathcal{B}_{m}^{i, j}$ es isomorfa a $\mathcal{A}_{m-1}$ y por proposición 2.2.8 $\mathcal{A}_{m-1}$ contiene una trayectoria hamiltoniana con vértices terminales $I_{m-1} * D_{m-1}$ y $I_{m-1} * D_{m-1}^{\prime}$. Por lo tanto $\mathcal{B}_{m}^{i, j}$ contiene una trayectoria hamiltoniana $K_{m}^{i, j}$ con extremos $\beta_{i, j}^{-1}\left(I_{m-1} * D_{m-1}\right)$ y $\beta_{i, j}^{-1}\left(I_{m-1} * D_{m-1}^{\prime}\right)$. Para finalizar la prueba mostraremos como conectar $K_{m}^{m, m}, K_{m}^{1, m}, K_{m}^{m, 1}, K_{m}^{1,1}$, para formar una trayectoria hamiltoniana $K_{m}$ de $\mathcal{B}_{m}$, con extremos $S_{m}=$ $\beta_{m, m}^{-1}\left(I_{m-1} * D_{m-1}\right)$ y $S_{m}^{\prime}=\beta_{1,1}^{-1}\left(I_{m-1} * D_{m-1}\right)$. 
Como $\theta^{-1}\left(I_{m-1}\right)=\theta\left(I_{m-1}\right)=I_{m-1}$ y $\theta^{-1}\left(D_{m-1}\right)=\theta\left(D_{m-1}\right)=D_{m-1}$ entonces $\beta_{m, m}^{-1}\left(I_{m-1} * D_{m-1}^{\prime}\right)=I_{m-1} * u_{m} v_{m} * D_{m-1}^{\prime}$ es adyacente a $I_{m-1} *$ $u_{1} v_{m} * D_{m-1}^{\prime}=\beta_{1, m}^{-1}\left(I_{m-1} * D_{m-1}^{\prime}\right)$. De manera análoga $\beta_{1, m}^{-1}\left(I_{m-1} * D_{m-1}\right)$ es adyacente a $\beta_{m, 1}^{-1}\left(I_{m-1} * D_{m-1}\right)$ y también $\beta_{m, 1}^{-1}\left(I_{m-1} * D_{m-1}^{\prime}\right)$ es adyacente a $\beta_{1,1}^{-1}\left(I_{m-1} * D_{m-1}^{\prime}\right)$.

Teorema 2.2.12 Si $n \geq 3$, entonces $T\left(P_{n}\right)$ es hamiltoniana.

\section{DEMOSTRACIÓN}

Como $n$ es un número impar o par, entonces consideraremos los dos casos.

\section{CASO 1. Sea $n=2 m+1$, con $m \geq 1$.}

Para $m=1$, tenemos de la figura 2.1 que $T\left(P_{3}\right)$ es hamiltoniana. Sea $m \geq 2$, para $k=0,1, \ldots, 2 m$ definimos $A^{k}$ como el conjunto de trayectorias generadoras planas de $T\left(P_{2 m+1}\right)$, con punto medio $w_{k+1}$ y $\mathcal{A}^{k}$ como la subgráfica de $T\left(P_{2 m+1}\right)$, inducida por $A^{k}$.

Sea $\lambda: P_{2 m+1} \longrightarrow P_{2 m+1}$ la función dada por $\lambda\left(w_{t}\right)=w_{t+1}$. Como $A^{k}$ es obtenida de $A^{m}$ por la rotación definida por $\lambda^{k-m}$ entonces $\mathcal{A}^{k}$ es isomorfa a $\mathcal{A}^{m}$. Como $\mathcal{A}^{m}=\mathcal{A}_{m}$ por proposición 2.2.8, $\mathcal{A}^{m}$ contiene una trayectoria hamiltoniana $J_{m}$ con extremos $I_{m} * D_{m}$ y $I_{m} * D_{m}^{\prime}$. Por lo tanto $\mathcal{A}^{k}$ contiene una trayectoria hamiltoniana $J_{k}$ con extremos $\lambda^{k-m}\left(I_{m} * D_{m}\right)$ y $\lambda^{k-m}\left(I_{m} * D_{m}^{\prime}\right)$. Para terminar el primer caso de la prueba, mostraremos como concatenar $J_{0}, J_{1}, \ldots J_{2 m}$ para obtener un ciclo hamiltoniano de $T\left(P_{2 m+1}\right)$. 
Definimos a $\lambda^{k-m}\left(I_{m} * D_{m}\right)$ como el extremo izquierdo de $J_{k}$ y a $\lambda^{k-m}\left(I_{m} *\right.$ $D_{m}^{\prime}$ ) como el extremo derecho de $J_{k}$.

Como $\left(I_{m} * D_{m}^{\prime}-w_{m+1} w_{2 m+1}\right)+w_{2 m+1} w_{1}=\lambda^{m+1}\left(I_{m} * D_{m}\right)$, entonces el extremo derecho de $J_{m}$, el cual es $I_{m} * D_{m}^{\prime}$, es adyacente a $\lambda^{m+1}\left(I_{m} * D_{m}\right)$ el cual es el extremo izquierdo de $J_{2 m+1}$ (Ver figura 2.7). Afirmamos que para toda $t$, el extremo derecho de $J_{t}$ es adyacente al extremo izquierdo de $J_{t+(m+1)}$ y por lo tanto $J_{m}, J_{2 m+1}=J_{0}, J_{3 m+2}=J_{m+1}, J_{4 m+3}=J_{1}, \ldots, J_{(2 m+1) m+2 m}=$ $J_{2 m}$ pueden ser concatenados en este orden para formar un ciclo hamiltoniano en $T\left(P_{2 m+1}\right)$.
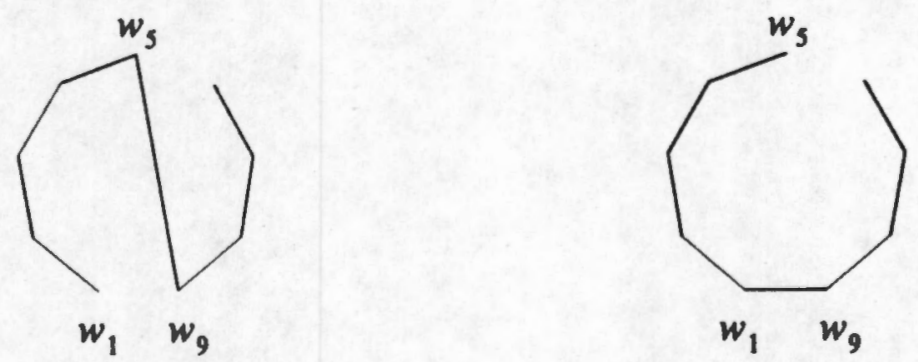

$$
\lambda^{0}\left(I_{4} * D_{4}^{\prime}\right) \text { y } \lambda^{5}\left(I_{4} * D_{4}\right)
$$

Figura 2.7

CASO 2. Sea $n=2 m, m \geq 2$.

Si $m=2$ entonces la figura 2.1 muestra que $T\left(P_{4}\right)$ es hamiltoniana. Supongamos entonces que $m \geq 3$.

Para $k=1,2, \ldots, 2 m$, sea $B^{k}$ el conjunto de trayectorias generadoras planas $T$ de $T\left(P_{2 m}\right)$ con arista media $w_{i} w_{j}$ (con $i \in\{k, k-m+1\}$ y $j \in$ $\{k+1, k+m\})$ tales que $V\left(T_{m}\right)=\left[w_{k-m+1}, w_{k}\right]$ y $V\left(T_{m+1}\right)=\left[w_{k+1}, w_{k+m}\right]$. 
Por simetría $B^{k}=B^{k+m}$.

Para $k=m, m+1, \ldots, 2 m-1$, sean $\mathcal{B}^{k}$ la subgráfica de $T\left(P_{2 m}\right)$ inducida por $B^{k}$ y $\mu: P_{2 m} \rightarrow P_{2 m}$ la función dada por $\mu\left(w_{t}\right)=w_{t+1}$. Como $B^{k}$ es obtenida de $B^{m}$, por la rotación definida por $\mu^{k-m}$ entonces $\mathcal{B}^{k}$ es isomorfa a $\mathcal{B}^{m}$. Como $\mathcal{B}^{m}=\mathcal{B}_{m}$, por proposición $2.2 .11, \mathcal{B}^{m}$ contiene una trayectoria hamiltoniana $K_{m}$ con extremos $S_{m}$ y $S_{m}^{\prime}$; por lo tanto $\mathcal{B}^{k}$ contiene una trayectoria hamiltoniana $K^{k}$ con extremos $\mu^{k-m}\left(S_{m}\right)$ y $\mu^{k-m}\left(S_{m}^{\prime}\right)$.

Como todas las trayectorias $\mu^{k-m}\left(S_{m}\right)$ y todas las trayectorias $\mu^{k-m}\left(S_{m}^{\prime}\right)$ son obtenidas del ciclo $C=w_{1}, w_{2}, \ldots, w_{2 m}$ quitándole una arista, entonces todas ellas son adyacentes por pares en $T\left(P_{2 m}\right)$. Por lo tanto $K_{m}, K_{m+1}, \ldots$, $K_{2 m-1}$ pueden ser conectadas (de muchas formas), para obtener un ciclo $Q_{2 m}$ de $T\left(P_{2 m}\right)$ que contiene todos los elementos de $B^{m} \cup B^{m+1} \cup \ldots \cup B^{2 m-1}$. Demostraremos ahora que $Q_{2 m}$ es un ciclo hamiltoniano de $T\left(P_{2 m}\right)$.

Sea $T$ un árbol generador plano de $T\left(P_{2 m}\right)$ con arista media e. Por teorema 2.2.3, los primeros $m$-vértices de $T$ son puntos consecutivos de $P_{2 m}$; por lo tanto los últimos $m$ vértices de $T$ también son consecutivos en $P_{2 m}$. Por lo tanto existe un entero $s$, con $1 \leq s \leq 2 m$ tal que $T$ - e está formada por una subtrayectoria izquierda $T_{I}$, con conjunto de vértices $\left[w_{s-m+1}, w_{s}\right]$, y una subtrayectoria derecha $T_{D}$, con conjunto de vértices $\left[w_{s+1}, w_{s+m}\right]$.

Como $e$ es una arista media de $T$, entonces $e$ tiene un extremo en $T_{I}$ y el otro en $T_{D}$; sea $t$, con $1 \leq t \leq m$, tal que $w_{s+t}$ es el extremo de $e$ en $T_{D}$. Como $P^{\prime}=V\left(T_{I}\right) \cup\left\{w_{s+t}\right\}$ es un conjunto de puntos en posición convexa, entonces $T_{I}+e$ es una trayectoria generadora plana de $P^{\prime}$ y $e$ es una arista terminal de $T_{I}+e$, por lo tanto $e$ pertenece a la frontera de $C H\left(P^{\prime}\right)$. 
Observe que $w_{s-m+1}$ y $w_{s}$ son dos puntos en $P^{\prime}$ los cuales son adyacentes a $w_{s+t}$ en la frontera de $C H\left(P^{\prime}\right)$, por lo tanto uno de estos vértices es extremo de $e$ en $T_{I}$.

De manera análoga el extremo de $e$ en $T_{D}$ es uno de los vértices $w_{s+1}$ o $w_{s+m}$; por lo tanto $e \in\left\{w_{s} w_{s+1}, w_{s} w_{s+m}, w_{s-m} w_{s+1}, w_{s-m+1} w_{s+m}\right\}$. Como $T_{I}$ tiene a $\left[w_{s-m+1}, w_{s}\right]$ como conjunto de vértices y $T_{D}$ tiene a $\left[w_{s+1}, w_{s+m}\right]$ como conjunto de vértices, entonces $T=T_{I} * e * T_{D} \in B^{s}=B^{s+m}$. 


\section{Capítulo 3}

\section{Existencia de Árboles Generadores Planos.}

\subsection{Introducción}

Uno de los problemas abiertos para gráficas geométricas es encontrar condiciones necesarias y suficientes en dichas gráficas para que éstas contengan un árbol generador plano.

G. Károlyi, J. Pach y G. Tóth en [9], demostraron que para cualquier coloración de las aristas, con das colores, de la gráfica geométrica completa, existe un árbol generador plano tal que todas sus aristas tienen asignado el mismo color; equivalentemente, dada una gráfica geométrica cualquiera, ella o su complemento contiene un árbol generador plano. En esta dirección, caracterizaremos en este capítulo aquellos árboles generadores planos cuyos complementos contienen un árbol generador plano (en [8]. Hernando, C. resolvió el problema en el caso convexo) y daremos una condición suficiente para que en el complemento de una gráfica geométrica exista un árbol generador plano.

\subsection{Estrellas y Gusanos.}

Para establecer el primer resultado daremos las siguientes definiciones y probaremos el siguiente lema.

Sea $P$ una colección de $n$ puntos en el plano en posición general. 
Si $T$ es un árbol generador plano de $K(P)$ y $e$ es una arista de $T$, entonces $L[e]$ denotará a la recta que pasa a través de e. A $L[e]$ le llamaremos la extensión de e. Ver figura 3.1.

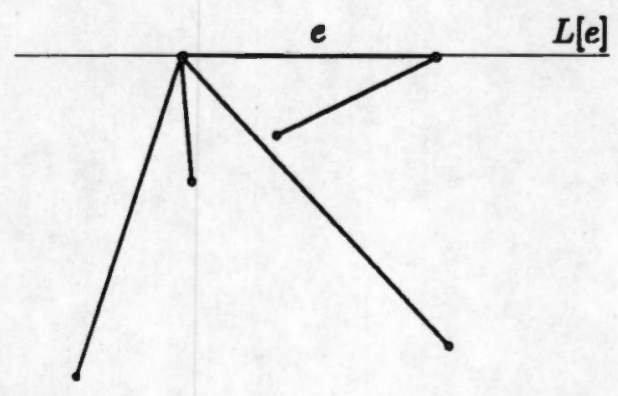

Figura 3.1

Observe que si $T$ es un árbol generador plano de $K(P)$ y e es una arista en la frontera de $C H(P)$ entonces $T$ está totalmente contenido en uno de los semiplanos cerrados definidos por la extensión de $e$.

Si $T$ es un árbol generador plano de $K(P)$ decimos que $T$ es un gusano si:

i) La subgráfica inducida por todos los vértices no-terminales de $T$, es una trayectoria que está totalmente contenida en la frontera de $C H(P)$.

ii) Para cualquier arista $e$ de $T$, su extensión $L[e]$ es tal que $L[e] \cap T=\{e\}$. La figura 3.2 muestra un ejemplo

Si $T$ es una trayectoria generadora plana de $K(P)$, decimos que $T$ es un caracol si: 


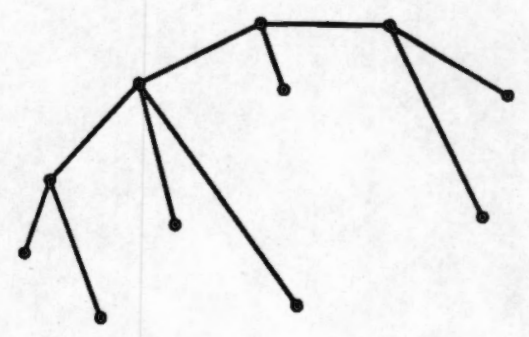

Figura 3.2

i) Todos los vértices y las aristas de $T$ pertenecen a la frontera de $C H(P)$, excepto un vértice terminal y una arista terminal.

ii) Si $e_{1}, e_{2}$ son las aristas terminales de $T$ y $e_{1}$ no está en la frontera de $C H(P)$ entonces $L\left[e_{1}\right]$ intersecta a $T$ en algún punto de $e_{2}$.

Ver ejemplo en la figura 3.3.

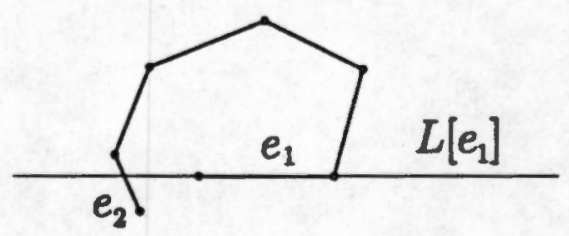

Figura 3.3

Lema 3.2.1 Sean $P$ una colección de puntos en el plano en posición general con al menos 5 elementos y $T$ un árbol generador plano de $K(P)$.

Si $T$ no es un gusano, una estrella o un caracol entonces existe un vértice terminal $x$ de $T$ tal que $T-x$ no es gusano ni estrella. 


\section{DEMOSTRACIÓN}

Si en $T$ existe una trayectoria $z_{1}, z_{2}, z_{3}, z_{4}$ para la cual, $L\left[z_{2} z_{3}\right]$ es tal que $z_{1}$ pertenece a uno de los semiplanos abiertos definidos por $L\left[z_{2} z_{3}\right]$ y $z_{4}$ pertenece al otro semiplano (ver figura 3.4), entonces como $|P| \geq 5$, existe un vértice terminal $x$ en $T$ tal que $x \neq z_{1}$ y $x \neq z_{4}$. Es claro que $T-x$ no es gusano ni estrella, ya que $z_{2}$ y $z_{3}$ son dos vértices no-terminales que no pertenecen a la frontera de $C H(P-x)$.

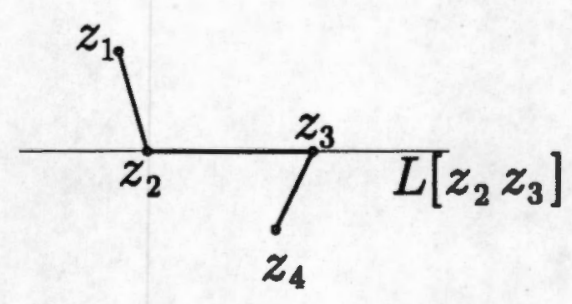

Figura 3.4

Supongamos entonces que dicha trayectoria no existe. Sea $U=u_{1}, u_{2}, \ldots, u_{\ell}$ una trayectoria de longitud máxima en $T$. Es claro que $\ell \geq 3$. Sean $v \in V(T) \backslash V(U), u_{j} \in V(U)$ y $\mathcal{V}=v_{1}, v_{2}, \ldots, v_{m}$ la trayectoria en $T$ que va de $v=v_{1}$ a $u_{j}=v_{m}$. Como $U$ es de longitud máxima, por lo tanto $2 \leq j \leq \ell-1$, (ver figura 3.5). Podemos suponer que $v_{m}$ es el único vértice de $\mathcal{V}$ que pertenece a $U$, de otra forma podemos definir $v_{m}$ como el primer vértice de $U$ que aparece en $\mathcal{V}$, al recorrer $\mathcal{V}$ de $v$ a $u_{j}$.

Si $m \geq 3$ entonces $j \notin\{1,2, \ell-1, \ell\}$. Como las aristas $u_{j} u_{j-1}, u_{j} u_{j+1}$ y $u_{j} v_{m-1}$ forman una estrella entonces la extensión de alguna de estas tres aristas es tal que deja a los otros dos vértices en semiplanos distintos. Por 


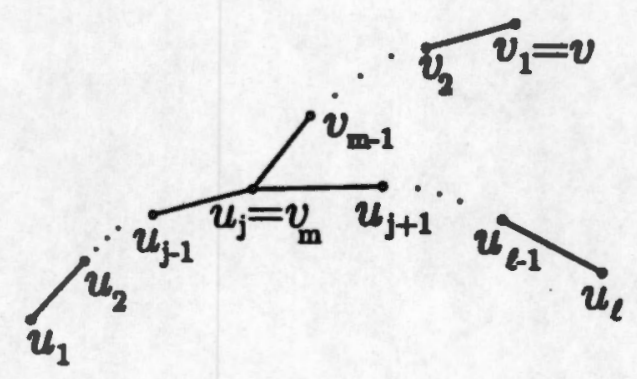

Figura 3.5

lo tanto, sin pérdida de generalidad, podemos suponer que la extensión $L\left[v_{m-1} u_{j}\right]$ es tal que si $H_{1}$ y $H_{2}$ son los semiplanos abiertos definidos por la recta entonces $u_{j-1} \in H_{1}$ y $u_{j+1} \in H_{2}$. Por lo tanto $v_{m-2} \notin H_{1}$ y $v_{m-2} \notin H_{2}$, lo cual es imposible. Esto implica que $m \leq 2$ y por lo tanto $v$ es adyacente a $u_{j}$, con $2 \leq j \leq \ell-1$. Tenemos entonces que $u_{2}, u_{3}, \ldots, u_{\ell-1}$ son todos los vértices no-terminales de $T$ e inducen una trayectoria en $T$.

Si la trayectoria inducida por los vértices $u_{2}, \ldots u_{\ell-1}$ está contenida en la frontera de $C H(P)$, entonces como $T$ no es un gusano, existe $e \in E(T)$ tal que $L[e]$ intersecta a $T$ en puntos que no están en $e$. Si $L[e]$ intersecta a $T$ en un punto que pertenece a una arista $e^{\prime}$, que no es arista terminal de $T$ cntonces existe un vértice terminal $x$, tal que $x$ no es extremo de $e$ y claramente $T-x$ no es gusano ni estrella. Si $L[e]$ intersecta a $T$ sólo en puntos que pertenecen a aristas terminales entonces sea $e_{1}$ una de dichas aristas terminales. Como $T$ no es un caracol entonces $e$ y $e_{1}$ no pueden ser las únicas aristas terminales en $T$, por lo tanto existe un vértice terminal $x$ de $T$ que no es extremo de $e$ ni de $e_{1}$ y en consecuencia $T-x$ no es gusano ni estrella.

Supongamos entonces que en $T$ existe una arista $e=u_{i-1} u_{i}$ con $3 \leq i \leq$ 
$\ell-1$, tal que $e$ no está en la frontera de $C H(P)$. Sean $L[e]$, la extensión de $e$ y $H_{1}, H_{2}$ los semiplanos abiertos definidos por la extensión. Sin pérdida de generalidad $u_{i-2}, u_{i+1} \in H_{1}$. Como $e$ no está en la frontera de $C H(P)$ entonces existe un vértice $w \in H_{2}$.

Si $L[e] \cap T=\{e\}$, entonces sea $W$ la trayectoria $w=w_{1}, w_{2}, \ldots, w_{s}=u_{i}$. Podemos suponer que $u_{i-1}$ no pertenece a $W$. Por lo tanto los vértices $w_{1}, w_{2}, \ldots, w_{s-1}$ pertenecen a $H_{2}$, pero entonces $u_{i-2}, u_{i-1}, u_{i}=w_{s}, w_{s-1}$ es una trayectoria tal que $u_{i-2} \in H_{1}$ y $w_{s-1} \in H_{2}$, lo cual es imposible. Así, $L[e]$ intersecta a $T$ en un punto de $e^{\prime} \in E(T), e^{\prime} \neq e$. Luego existe un vértice terminal $x$, que no es extremo de $e^{\prime}$ ni de $e$, tal que $T-x$ no es gusano ni estrella.

Teorema 3.2.2 Sea $T$ un árbol generador plano de $K(P)$. $T$ no es un gusano o una estrella si y sólo si $T^{c}$ contiene un árbol generador plano.

\section{DEMOSTRACIÓN}

Supongamos que $T$ no es un gusano ni una estrella.

Probaremos por inducción sobre $|P|$, que $T^{c}$ contiene un árbol generador plano.

Suponga el teorema válido siempre que $|P|=k$. Sea $P$ un conjunto con $n=k+1$ elementos.

Consideremos los siguientes dos casos. 
CASO 1. Si $T$ es un caracol, entonces $T$ es la trayectoria $v_{1}, v_{2}, \ldots, v_{n}$. Sin pérdida de generalidad $L\left[v_{1} v_{2}\right]$ intersecta a $T$ en algún punto de $v_{n-1} v_{n}$. Por lo tanto la gráfica cuyo conjunto de vértices es $\left\{v_{1}, v_{2}, \ldots, v_{n}\right\}$ y cuyo conjunto de aristas es $\left\{v_{1} v_{3}, v_{1} v_{4}, \ldots, v_{1} v_{n}, v_{n} v_{2}\right\}$, es un árbol generador plano $R$ en $T^{c}$ (ver figura 3.6).

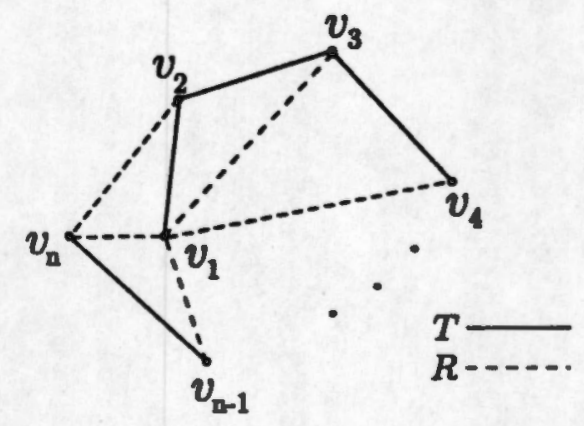

Figura 3.6

CASO 2. Supongamos que $T$ no es un caracol.

Entonces por el lèma 3.2.1, existe un vértice terminal $x$ de $T$ tal que $T-x$ no es un gusano o una estrella. Por hipótesis de inducción $(T-x)^{c}$ contiene un árbol generador plano $T^{*}$.

Sea $D$ una triangulación de $P-x$ que contenga todas las aristas de $T^{*}$.

Si $x$ está en el interior de $C H(P-x)$ entonces, como $D$ es una triangulación de $P-x, x$ está contenido en una cara triangular uvw de $D$. Sin pérdida de generalidad, $x$ es adyacente a $u$ en $T^{c}$. Es claro que $T^{*}$, junto con el vértice $x$ y la arista $x u$ es un árbol generador plano de $T^{c}$. 
Si $x$ no está en el interior de $C H(P-x)$, entonces $x$ es visible desde al menos dos vértices de la frontera de $C H(P-x)$. Sea $u$ un vértice de la frontera de $C H(P-x)$ desde el cual $x$ es visible y tal que $u$ y $x$ son adyacentes en $T^{c}$ (esto es posible pues $x$ es de grado 1 en $T$ ). Agregando a $T^{*}$ el vértice $x$ y la arista $x u$ obtenemos un árbol generador plano de $T^{c}$.

Demostraremos ahora que si $T$ es una estrella o un gusano entonces $T^{c}$ no contiene árboles generadores planos.

Si $T$ es una estrella, entonces es claro que el resultado se cumple.

Sea $T$ un gusano que no es estrella y supongamos que $T^{c}$ contiene al menos un árbol generador plano.

Elijamos $T$ como el mínimo gusano tal que $T^{\mathcal{C}}$ contiene un árbol generador plano $R$. Sean $u_{1}, u_{2}, \ldots, u_{m}$ los vértices no-terminales de $T$, ordenados en el sentido en que se mueven las manecillas del reloj.

Demostraremos primero que dos vértices no-terminales de $T$ no son adyacentes en $R$. Supongamos que esto no ocurre. Sean $u_{j}$ y $u_{k}$ dos vértices no-terminales de $T$ adyacentes en $R$, con $1 \leq j<k \leq m$. Definimos $T^{*}$ y $R^{*}$ como sigue:

i) Los vértices de $T^{*}$ son los vértices no-terminales de $T$ que se encuentran entre $u_{j}$ y $u_{k}$, incluyendo a $u_{j} \mathrm{y}$ a $u_{k} \mathrm{y}$ todos los vértices terminales de $T$ tales que las aristas terminales incidentes a ellos no intersectan la arista $u_{j} u_{k}$, excepto aquellos vértices terminales adyacentes a $u_{j}$ y a $u_{k}$. Dos vértices de $T^{*}$ son adyacentes si y sólo si son adyacentes en $T$. 
ii) El conjunto de vértices de $R^{*}$ es el mismo de $T^{*}$, y dos vértices de $R^{*}$ son adyacentes si y sólo si son adyacentes en $R$.

Es claro que $T^{*}$ es un gusano, que $\left|V\left(T^{*}\right)\right|<|V(T)|$ y que $R^{*}$ es un árbol generador plano de $\left(T^{*}\right)^{c}$, pero esto contradice la elección de $T$; por lo tanto dos vértices no-terminales de $T$, no son adyacentes en $R$, es decir, si $w$ es un vértice no-terminal de $T$ entonces $w$ es adyacente a un vértice terminal de $T$ en $R$.

Sea $\Gamma_{T}(v)$ la vecindad de $v$ en $T$. Como $u_{1}$ es un vértice no-terminal de $T$, entonces $u_{1}$ es adyacente en $R$, a un vértice terminal de $v_{1}$ de $T$, por lo tanto $v_{1} \in \Gamma_{T}\left(u_{j_{1}}\right)$, para alguna $1<j_{1} \leq m$. Ya que $u_{2}$ es un vértice no-terminal de $T, u_{2}$ es adyacente en $R$ a un vértice terminal $v_{2}$ de $T$. Probaremos que $v_{2} \in \Gamma_{T}\left(u_{j_{2}}\right)$, con $2<j_{2} \leq m$. Es obvio que $j_{2} \neq 2$. Supongamos que $j_{2}=1$. Como $u_{1}$ y $v_{1}$ son adyacentes en $R$ y $v_{1} \in \Gamma_{T}\left(u_{j_{1}}\right)$, entonces la extensión $L\left[u_{j_{1}} v_{1}\right]$ intersecta a $W$ en algún punto que no está en $u_{j_{1}} v_{1}$ o bien la extensión $L\left[u_{1} v_{2}\right]$ intersecta a $W$ en algún punto que no está en $u_{1} v_{2}$ pero esto no es posible pues $T$ es un gusano. Este argumento puede ser repetido de manera similar. Supongamos que para $i, 1 \leq 1 \leq k$, los vértices $u_{i} \mathrm{y}$ $v_{i}$ son adyacentes en $R$ con $v_{i} \in \Gamma_{T}\left(u_{j_{i}}\right)$ y $i<j_{i} \leq m$. Supongamos que $u_{k+1}$ es adyacente en $R$ a un vértice terminal $v_{k+1}$ de $T$. Probaremos que $v_{k+1} \in \Gamma_{T}\left(u_{j_{k+1}}\right)$ con $k+1<j_{k+1} \leq m$. Como en el argumento previo $j_{k+1} \neq k+1$.

Supongamos que $1 \leq j_{k+1}<k+1$. Como $u_{k}, v_{k}$ son adyacentes en $R$ y $v_{k} \in \Gamma_{T}\left(u_{j_{k}}\right)$, con $k<j_{k} \leq m$, entonces $L\left[u_{j_{k}} v_{k}\right]$ intersecta a $T$ en algún punto que no está en $u_{j_{k}} v_{k}$ o bien $L\left[u_{j_{k+1}}, v_{k+1}\right]$ intersecta a $T$ en algún punto 
que no está en $u_{j_{k+1}} v_{k+1}$, (ver figura 3.7), pero ésto no es posible, pues $T$ es un gusano. Por lo tanto $u_{k+1}$ es adyacente en $R$ a $v_{k+1} \in T$ y $v_{k+1} \in \Gamma\left(u_{j_{k+1}}\right)$ con $k+1<j_{k+1} \leq m$. En particular, si $i=m$, entonces $u_{m}$ es adyacente a $v_{m}$ y $v_{m} \in \Gamma_{T}\left(u_{j_{m}}\right)$ con $m<j_{m} \leq m$. Pero esta última desigualdad es contradictoria y por lo tanto $T^{c}$ no contiene árboles generadores planos.
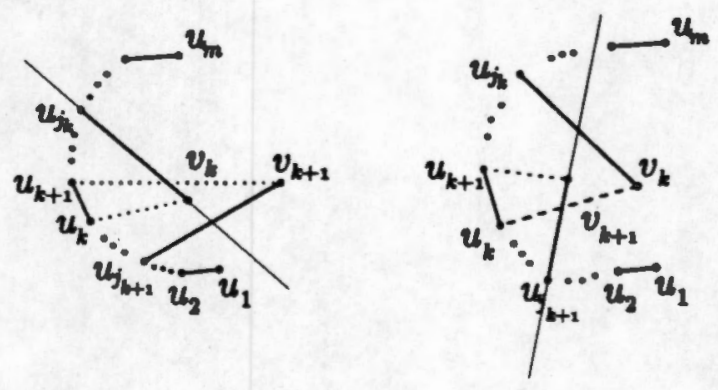

Figura 3.7

\subsection{Triángulos Vacíos y Ciclos de Rectas.}

Sean $P$ una colección de $n$ puntos en posición general en el plano, $G$ una gráfica geométrica sobre $P$ y $x, y, z \in P$. Decimos que $x y z$ es un triángulo de $G$ si al menos dos de las tres aristas posibles entre los tres vértices pertenecen a $E(G)$. Decimos que $x y z$ es un triángulo vacío de $G$, si $x y z$ es un triángulo de $G$ tal que en su interior no existen elementos de $P$. En la figura 3.8, $a b c$ no es un triángulo vacío, $b c d$ si lo es y éstos son los únicos triángulos de $G$. Denotaremos por $s(G)$, al número de triángulos vacíos de $G$. En esta sección demostraremos que si $G$ es una gráfica geométrica con a lo más $n-3$ triángulos vacíos entonces existe un árbol generador plano en $G^{c}$. 


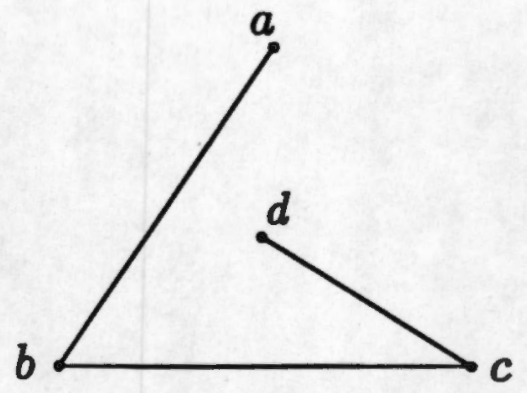

Figura 3.8

Daremos primero las siguientes definiciones y lemas.

Para cada recta orientada $L$ denotamos por $L^{-}$, al conjunto de puntos de $P$ que están sobre o a la izquierda de $L$ y por $L^{+}$a los puntos que están sobre o a la derecha de $L$. Sean $v_{1}$ un punto en $P$ y $L=L_{1}$ una recta orientada que pasa por $v_{1}$. Rotemos $L$, con eje en $v_{1}$, en el sentido de las manecillas del reloj, hasta alcanzar un punto $v_{2}$ de $P$. Continuemos rotando $L$ en el mismo sentido, ahora con eje en $v_{2}$, hasta al canzar un punto $v_{3}$ de $P$ y así sucesivamente. Al conjunto infinito de rectas obtenido de esta manera, a partir de $L_{1}$ lo llamaremos el ciclo de $L_{1}$ y lo denotamos por $\mathcal{C}\left(L_{1}\right)$. Para $i \geq 1$, sea $L\left(v_{i}, v_{i+1}\right)$ la recta en $\mathcal{C}\left(L_{1}\right)$ que pasa por los puntos $v_{i}$ y $v_{i+1}$. Como $P$ es un conjunto finito de puntos en posición general, entonces existe $\alpha>0$ tal que para cada $i>1$, el ángulo entre las rectas $L\left(v_{i-1}, v_{i}\right)$ y $L\left(v_{i}, v_{i+1}\right)$ es por lo menos $\alpha$. Por lo tanto existe un entero $k$ tal que $v_{k}=v_{1}$ y $L_{k}=L_{1}$ (misma recta y misma orientación), esto justifica el nombre de ciclo de rectas dado al conjunto $\mathcal{C}\left(L_{1}\right)$. Para $i=2,3, \ldots, k-1$, sea $L_{i}$ una recta cualquiera en $\mathcal{C}\left(L_{1}\right)$ entre $L\left(v_{i-1}, v_{i}\right)$ y $L\left(v_{i}, v_{i+1}\right)$, ver figura 3.9.

Los siguientes lemas dan la propiedad principal de los ciclos de rectas. 


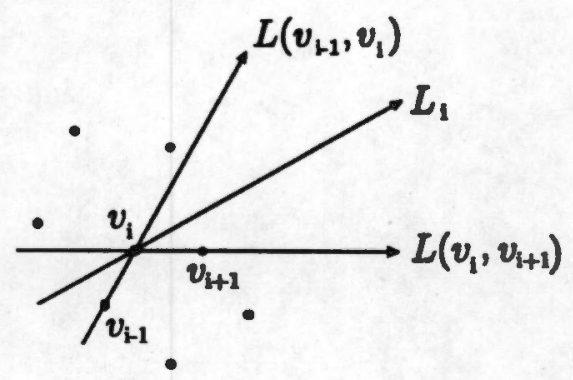

Figura 3.9

Lema 3.3.1 Para $i=2,3, \ldots, k,\left|L_{i}^{-}\right|=\left|L_{1}^{-}\right| y\left|L_{i}^{+}\right|=\left|L_{1}^{+}\right|$.

\section{DEMOSTRACIÓN}

Si $v_{i} \in L_{i-1}^{+}$, entonces $L_{i}^{-}=\left(L_{i-1}^{-} \cup\left\{v_{i}\right\}\right)-\left\{v_{i-1}\right\}$ y $L_{i}^{+}=L_{i-1}^{+}$. De manera análoga si $v_{i} \in L_{i-1}^{-}$entonces $\left.L_{i}^{+}=L_{i-1}^{+} \cup\left\{v_{i}\right\}\right)-\left\{v_{i-1}\right\}$ y $L_{i}^{-}=L_{i-1}^{-}$.

Lema 3.3.2 Para $i=2,3, \ldots, k,\left|L^{-}\left(v_{i-1}, v_{i}\right)\right|=\left|L_{i-1}^{-}\right|+1$ y $\left|L^{+}\left(v_{i-1}, v_{i}\right)\right|=$ $\left|L_{i-1}^{+}\right| \circ\left|L^{+}\left(v_{i-1}, v_{i}\right)\right|=\left|L_{i-1}^{+}\right|+1 y\left|L^{-}\left(v_{i-1}, v_{i}\right)\right|=\left|L_{i-1}^{-}\right|$.

\section{Demostración}

Si $v_{i} \in L_{i-1}^{+}$, entonces $L^{-}\left(v_{i-1}, v_{i}\right)=L_{i-1}^{-} \cup\left\{v_{i}\right\}$ y $L^{+}\left(v_{i-1}, v_{i}\right)=L_{i-1}^{+} ;$y si $v_{i} \in L_{i-1}^{-}$, entonces $L^{+}\left(v_{i-1}, v_{i}\right)=L_{i-1}^{+} \cup\left\{v_{i}\right\}$ y $L^{-}\left(v_{i-1}, v_{i}\right)=L_{i-1}^{-}$.

Lema 3.3.3 Sean $M$ y $L$ rectas orientadas que contienen cada una exactamento un punto de $P$. Si $\left|M^{-}\right|=\left|L^{-}\right| y\left|M^{+}\right|=\left|L^{+}\right|$entonces $\mathcal{C}(M)=\mathcal{C}(L)$. 


\section{Demostración}

El ciclo $\mathcal{C}(L)$ contiene una única recta $L^{\prime}$ paralela a $M$ con la misma orientación. Si $M \neq L^{\prime}$ podemos suponer sin pérdida de generalidad que $M$ está a la izquierda de $L^{\prime}$. Como $L^{\prime}$ contiene al menos un punto $w$ de $P$ (a lo más dos puntos de $P$ ), entonces por un lado $\left|M^{-}\right|<\left|L^{\prime-}\right|$ pues $M^{-} \subset L^{\prime-}$ y $w \notin M^{-}$y por lema 3.3.1, $\left|L^{\prime-}\right|=\left|L^{-}\right|=\left|M^{-}\right|$lo cual es imposible, por lo tanto $M=L^{\prime} \in \mathcal{C}(L)$. De manera análoga $L \in \mathcal{C}(M)$.

Para ciclos de rectas que dividen a $P$ equitativamente tenemos el siguiente lema.

Lema 3.3.4 Sea $P$ una colección de $n$ puntos en posición general. Si $L$ es una recta tal que $\left|L^{-}\right|=\left\lfloor\frac{n+1}{2}\right\rfloor y\left|L^{+}\right|=\left\lceil\frac{n+1}{2}\right\rceil$, entonces para cada $v \in P$ existe $M \in \mathcal{C}(L)$ tal que $v$ es el único punto de $P$ en $M$.

\section{DeMOSTRACIÓN}

Sea $R$ una recta que contenga a $v$ y $A$ y $B$ las rectas con orientaciones opuestas correspondientes. Si $\left|A^{-}\right|<\left\lfloor\frac{n+1}{2}\right\rfloor$ (y por lo tanto $\left|A^{+}\right|>\left\lceil\frac{n+1}{2}\right\rceil$ ), entonces $\left|B^{+}\right|<\left\lfloor\frac{n+1}{2}\right\rfloor$ y $\left|B^{-}\right|>\left\lceil\frac{n+1}{2}\right\rceil$. Al rotar $A$ hacia $B$ (siempre con eje en $v$ ) aumentamos o disminuimos el número de puntos de $P$, a la izquierda de la recta en una unidad cada vez que pasamos por punto de $P$. Como $\left|A^{-}\right|<\left\lfloor\frac{n+1}{2}\right\rfloor$ y $\left|B^{-}\right|>\left\lceil\frac{n+1}{2}\right\rceil$ entonces existe una recta orientada $D$, entre $A$ y $B$, que pasa por $v$ y tal que $\left|D^{-}\right|=\left\lfloor\frac{n+1}{2}\right\rfloor$ y $\left|D^{+}\right|=\left\lceil\frac{n+1}{2}\right\rceil$. Por el lema 3.3.3, $D \in \mathcal{C}(L)$. 
Teorema 3.3.5 Si $G$ es una gráfica geométrica sobre un conjunto $P$ de $n$ puntos en posición general en el plano y $s(G) \leq n-3$, entonces $G^{c}$ contiene al menos un árbol generador plano.

\section{Demostración}

Si $|P|=3$ o $|P|=4$ por inspección se verifica el resultado. Supongamos el teorema es válido siempre que $3 \leq|P|=k<n$.

Sean $P$ una colección con $n \geq 5$ puntos en posición general y $G$ una gráfica geométrica sobre $P$ tal que $s(G) \leq n-3$. Sea $v_{1}$ un punto de $P$, $L_{1}$ una recta orientada que pasa unicamente por $v_{1}$ tal que $\left|L_{1}^{-}\right|=\left\lceil\frac{n+1}{2}\right\rceil$ y $\left|L_{1}^{+}\right|=\left\lfloor\frac{n+1}{2}\right\rfloor$, y $\mathcal{C}\left(L_{1}\right)$ el ciclo de rectas de $L_{1}$.

Para cada $L_{i}$, con $L_{i} \in \mathcal{C}\left(L_{1}\right)$, definimos $G_{i}^{-}$y $G_{i}^{+}$como las subgráficas inducidas en $G$ por $L_{i}^{-}$y $L_{i}^{+}$respectivamente y para cada $L\left(v_{i-1}, v_{i}\right) \in \mathcal{C}\left(L_{1}\right)$ definimos $G^{-}\left(v_{i-1}, v_{i}\right)$ y $G^{+}\left(v_{i-1}, v_{i}\right)$ como las subgráficas inducidas en $G$ por $L^{-}\left(v_{i-1}, v_{i}\right)$ y $L^{+}\left(v_{i-1}, v_{i}\right)$ respectivamente. Como $n$ es un número impar o par, consideraremos los dos casos.

CASO 1. Si $n=2 \ell+1$, entonces $\left|L_{1}^{-}\right|=\ell+1=\left|L_{1}^{+}\right|$.

Calculando $s\left(G_{1}^{-}\right)$y $s\left(G_{1}^{+}\right)$tenemos los siguientes cuatro subcasos:

CASO 1i) Si $s\left(G_{1}^{-}\right) \leq\left|L_{1}^{-}\right|-3$ y $s\left(G_{1}^{+}\right) \leq\left|L_{1}^{+}\right|-3$, entonces por hipótesis de inducción, existen $T_{1}^{-}$y $T_{1}^{+}$, árboles generadores planos de $\left(G_{1}^{-}\right)^{c}$ y $\left(G_{1}^{+}\right)^{c}$. Es claro que $T_{1}^{-} \cup T_{1}^{+}$es un árbol generador plano de $G^{c}$ y el teorema se sigue. 
CASO 1ii) Si $s\left(G_{1}^{-}\right)>\left|L_{1}^{-}\right|-3$ y $s\left(G_{1}^{+}\right)>\left|L_{1}^{+}\right|-3$, entonces $s\left(G_{1}^{-}\right) \geq \ell-1$ y $s\left(G_{1}^{+}\right) \geq \ell-1$.

Como $2 \ell-2 \leq s\left(G_{1}^{-}\right)+s\left(G_{1}^{+}\right) \leq s(G) \leq 2 \ell-2$ entonces $s\left(G_{1}^{-}\right)=$ $s\left(G_{1}^{+}\right)=\ell-1$ y $s(G)=2 \ell-2$.

La última igualdad implica que $L_{1}$ no intersecta triángulos vacíos de G.

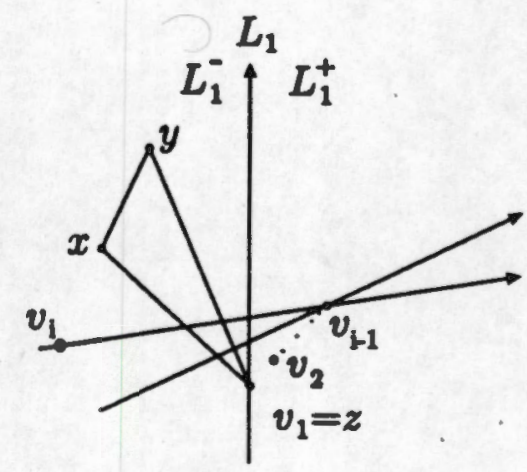

Figura 3.10

Probaremos que en $\mathcal{C}\left(L_{1}\right)$ existe una recta $L_{k}$ que intersecta algún triágulo vacío de $G$. Supongamos que esto no ocurre.

Sea $x y z$ un triángulo vacío de $G_{1}^{-}$. Sea $L_{2} \in \mathcal{C}\left(L_{1}\right)$ la recta que tiene a $v_{2}$ como eje. Si $v_{2} \in L_{1}^{-}$entonces $L_{2}^{-}=L_{1}^{-}$, por lo tanto $x y z$ es un triángulo vacío de $G_{2}^{-}$. Si $v_{2} \in L_{1}^{+}$entonces $L_{2}^{-}=\left(L_{1}^{-} \cup\left\{v_{2}\right\}\right) \backslash\left\{v_{1}\right\}$. Como $L_{1}$ y $L_{2}$ no intersectan triángulos vacíos de $G$ entonces ni $v_{1}$ ni $v_{2}$ son vértices de $x y z$ y por lo tanto $x y z$ es un triángulo vacío de $G_{2}^{-}$. De ambos casos podemos concluir que si $x y z$ es un triángulo vacío de $G_{1}^{-}$entonces $x y z$ es un triángulo vacío de $G_{2}^{-}$. 
Repitiendo el razonamiento anterior tenemos que $x y z$ es un triángulo vacío de $G_{i}^{-}$, para cualquier $L_{i} \in \mathcal{C}\left(L_{1}\right)$. En particular si $L_{m}$ es la única recta paralela a $L_{1}$ en $\mathcal{C}\left(L_{1}\right)$ entonces $x y z$ es un triángulo vacío de $G_{m}^{-}$.

Como $L_{1}$ y $L_{m}$ son paralelas y $\left|L_{1}^{-}\right|=\left|L_{1}^{+}\right|=\left|L_{m}^{-}\right|=\left|L_{m}^{+}\right|=\ell+1$ entonces $L_{1}$ y $L_{m}$ son la misma recta pero con orientación opuesta, por lo tanto $G_{m}^{-}=G_{1}^{+}$y $G_{m}^{+}=G_{1}^{-}$; de aquí que $x y z$ pertenece a $G_{1}^{+}$lo cual es imposible. Así existe una recta $L_{k} \in \mathcal{C}\left(L_{1}\right)$.tal que intersecta algún triángulo vacío de $G$.

Sean $L_{2}, L_{3}, \ldots, L_{k}$ las rectas en $\mathcal{C}\left(L_{1}\right)$ tales que $L_{2}, \ldots L_{k-1}$ no intersectan triángulos vacíos de $G$ y $L_{k}$ intersecta algún triángulo vacío de $G$. No es difícil ver que si $L_{1}$ es tal que $s\left(\overline{1}_{1}^{-}\right)=\ell-1=s\left(G_{1}^{+}=\right.$entonces $s\left(G_{i}^{-}\right)=\ell-1=s\left(G_{i}^{+}\right)$para toda $i=2,3, \ldots, k-1$. Por lo tanto sin pérdida de generalidad, $L_{k}=L_{2}, x y z$ es el triángulo vacío de $G$ que $L_{2}$ intersecta y $v_{1}$ es 'uno de los vértices del triángulo vacío, digamos $v_{1}=z$.

Como $v_{1} \neq v_{2}$ entonces $v_{2} \in L_{1}^{+}$o $v_{2} \in L_{1}^{-}$.

CASO 1 ii a) Si $v_{2} \in L_{1}^{+}$, entonces $x, y$ pertenecen a $L_{1}^{-}$y $s\left(G_{2}^{-}\right) \leq \ell-2=\left|L_{2}^{-}\right|-3$ y $s\left(G_{2}^{+}\right)=\ell-1=\left|L_{2}^{+}\right|-2$. Si $v_{3} \in L_{1}^{-}$entonces consideramos la recta $L\left(v_{2}, v_{3}\right)$, para la cual $\left|L^{-}\left(v_{2}, v_{3}\right)\right|=\left|L_{2}^{-}\right|=\left|L_{1}^{-}\right|=\ell+1$ y $\left|L^{+}\left(v_{2}, v_{3}\right)\right|=\left|L_{2}^{+}\right|+1=\ell+2$. Además $s\left(G^{-}\left(v_{2}, v_{3}\right)\right)=s\left(G_{2}^{-}\right) \leq$ $\ell-2$ y $s\left(G^{+}\left(v_{2}, v_{3}\right)\right)=s\left(G_{2}^{+}\right)=\ell-1$. Por lo tanto por hipótesis de inducción, existen $T^{-}\left(v_{2}, v_{3}\right)$ y $T^{+}\left(v_{2}, v_{3}\right)$ árboles generadores planos 
de $\left(G^{-}\left(v_{2}, v_{3}\right)\right)^{c}$ y de $\left(G^{+}\left(v_{2}, v_{3}\right)\right)^{c}$ respectivamente, luego $T^{-}\left(v_{2}, v_{3}\right) \cup$ $T^{+}\left(v_{2}, v_{3}\right)$ contiene un árbol generador plano de $G^{c}$.

Supongamos que $v_{3} \in L_{1}^{+}$. Como $x, y \in L_{1}^{-}$entonces por el lema 3.3.4, existe al menos una recta en $C\left(L_{1}\right)$ tal que su eje de rotación pertenece a $L_{1}^{-}$.

Sea $i$, la mínima $i$ para la cual $v_{i}$, el eje de $L_{i}$, pertenece a $L_{1}^{-}$, es decir $L_{2}, L_{3}, \ldots, L_{i-1}$, son tales que $L_{1}^{+}=L_{2}^{+}=\cdots=L_{i-1}^{+}$y $v_{2}, v_{3} \ldots v_{i-1} \in$ $L_{1}^{+}$y $L_{i}$ es tal que $v_{i} \in L_{1}^{-}$.

Consideremos la recta $L\left(v_{i-1}, v_{i}\right)$ de $\mathcal{C}\left(L_{1}\right)$ (ver figura 3.10), por lo tanto $\left|L^{-}\left(v_{i-1}, v_{i}\right)\right|=\left|L_{i-1}^{-}\right|,\left|L^{+}\left(v_{i-1}, v_{i}\right)\right|=\left|L_{i-1}^{+}\right|+1 \mathrm{y}$

$$
\begin{aligned}
s\left(G^{-}\left(v_{i-1}, v_{i}\right)\right) & =s\left(G_{i-1}^{-}\right) \leq s\left(G_{2}^{-}\right) \leq \ell-2=\left|L_{2}^{-}\right|-3 \\
& =\left|L_{i}^{-}\right|-3=\left|L^{-}\left(v_{i-1}, v_{i}\right)\right|-3 .
\end{aligned}
$$

Además

$$
\begin{aligned}
s\left(G^{+}\left(v_{i-1}, v_{i}\right)\right) & =s\left(G_{i-1}^{+}\right)=s\left(G_{2}^{+}\right)=\ell-1=\left|L_{2}^{+}\right|-2 \\
& =\left|L_{i-1}^{+}\right|-2=\left|L^{+}\left(v_{i-1}, v_{i}\right)\right|-3
\end{aligned}
$$

Por lo tanto, por hipótesis de inducción, existen $T^{-}\left(v_{i-1}, v_{i}\right)$ y $T^{+}\left(v_{i-1}, v_{i}\right)$ árboles generadores planos de $\left(G^{-}\left(v_{i-1}, v_{i}\right)\right)^{c}$ y $\left(G^{+}\left(v_{i-1}, v_{i}\right)\right)^{c}$ respectivamente. Es claro que $T^{-}\left(v_{i-1}, v_{i}\right) \cup T^{+}\left(v_{i-1}, v_{i}\right)$ contiene un árbol generador plano de $G^{c}$.

CASO $1 i i$ b) Si $v_{2} \in L_{1}^{-}$, la demostración es análoga al caso 1 ii a). 
CASO 1iii) Si $s\left(G_{1}^{-}\right) \leq\left|L_{1}^{-}\right|-3$ y $s\left(G_{1}^{+}>\left|L_{1}^{+}\right|-3\right.$, sea $L_{m}$ la única recta paralela a $L_{1}$ con orientación opuesta a $L_{1}$ en $C\left(L_{1}\right)$. Como $\left|L_{1}^{-}\right|=\left|L_{1}^{+}\right|=$ $\left|L_{m}^{-}\right|=\left|L_{m}^{+}\right|$entonces $L_{1}$ y $L_{m}$ son la misma recta, pero con orientación opuesta. Sean $L_{2}, L_{3}, \ldots, L_{m-1}$ la colección de rectas de $\mathcal{C}\left(L_{1}\right)$ que se encuentran entre $L_{1}$ y $L_{m}$. Si para toda $j$, con $1 \leq j \leq m s\left(G_{j}^{-}\right) \leq$ $\left|L_{j}^{-}\right|-3$ y $s\left(G_{j}^{+}\right)>\left|L_{j}^{+}\right|-3$ entonces en particular, $s\left(G_{m}^{-}\right) \leq\left|L_{m}^{-}\right|-3$ y $s\left(G_{m}^{+}\right)>\left|L_{m}^{+}\right|-3$. Como $L_{1}$ y $L_{m}$ son iguales pero con orientación opuesta, tenemos que

$$
\ell-2=\left|L_{1}^{+}\right|-3<s\left(G_{1}^{+}\right)=s\left(G_{m}^{-}\right) \leq\left|L_{m}^{-}\right|-3=\ell-2
$$

lo cual es absurdo. Por lo tanto existe $i, 2 \leq i \leq m-1$, tal que

$$
s\left(G_{i}^{-}\right) \leq\left|L_{i}^{-}\right|-3 \text { y } s\left(G_{i}^{+}\right) \leq\left|L_{i}^{+}\right|-3
$$

o bien

$$
s\left(G_{i}^{-}\right)>\left|L_{i}^{-}\right|-3 \text { y } s\left(G_{i}^{+}\right)>\left|L_{i}^{+}\right|-3
$$

En cualquiera de ambos casos el teorema se sigue, pues el primero es el caso 1 i) y el segundo es el caso 1 ii).

CASO 1iv) Si $s\left(G_{1}^{-}\right)>\left|L_{1}^{-}\right|-3$ y $s\left(G_{1}^{+}\right) \leq\left|L_{1}^{+}\right|-3$, la demostración es análoga al caso 1 iii).

CASo 2. Si $n=2 \ell$, entonces $\left|L_{1}^{-}\right|=\ell+1 \mid$ y $\left|L_{1}^{+}\right|=\ell$. Calculando $s\left(G_{1}^{-}\right)$y $s\left(G_{1}^{+}\right)$tenemos los siguientes casos.

CASO 2i) Si $s\left(G_{1}^{-}\right) \leq\left|L_{1}^{-}\right|-3$ y $s\left(G_{1}^{+}\right) \leq\left|L_{1}^{+}\right|-3$ entonces por hipótesis de inducción existen $T_{1}^{-}$y $T_{1}^{+}$árboles generadores planos de $\left(G_{1}^{-}\right)^{c}$ y $\left(G_{1}^{+}\right)^{c}$ 
respectivamente. Es claro que $T_{1}^{-} \cup T_{1}^{+}$es un árbol generador plano de $G^{c}$ y el teorema se sigue.

CASO 2ii) Si $s\left(G_{1}^{-}\right)>\left|L_{1}^{-}\right|-3$ y $s\left(G_{1}^{+}\right)>\left|L_{1}^{+}\right|-3$ por lo tanto $s\left(G_{1}^{-}\right) \geq \ell-1$ y $s\left(G_{1}^{+}\right) \geq \ell-2$. Observe que si $s\left(G_{1}^{-}\right)=\ell-1$ entonces

$$
\ell-1+s\left(G_{1}^{+}\right)=s\left(G_{1}^{-}\right)+s\left(G_{1}^{+}\right) \leq s(G) \leq 2 \ell-3
$$

y así $s\left(G_{1}^{+}\right) \leq \ell-2$, pero como $\ell-2 \leq s\left(G_{1}^{+}\right)$tenemos que $s\left(G_{1}^{+}\right)=\ell-2$. Por otro lado, si $s\left(G_{1}^{-}\right)>\ell-1$ procediendo de manera análog̨ tenemos que $s\left(G_{1}^{+}\right)<\ell-2$, lo cual es absurdo pues $s\left(G_{1}^{+}\right) \geq \ell-2$. De aquí podemos concluir que $s\left(G_{1}^{-}\right)=\ell-1$ y $s\left(G_{1}^{+}\right)=\ell-2$ y en consecuencia $s(G)=2 \ell-3$. Por lo tanto $L_{1}$ es una recta que no intersecta triángulos vacíos de $G$. De manera análoga al caso 1 ii) tenemos que en $\mathcal{C}\left(L_{1}\right)$ existe una recta que intersecta al menos un triángulo vacío de $G$, así podemos suponer, sin pérdida de generalidad, que $L_{2}$ la recta que pasa por $v_{2}$, es dicha recta y que $x y z$ es el triángulo vacío de $G$ que $L_{2}$ intersecta. Podemos suponer además que $v_{1}$ es uno de los vértices del triángulo vacío, es decir $v_{1}=z$.

Como $v_{1} \neq v_{2}$, entonces $v_{2} \in L_{1}^{+}$o $v_{2} \in L_{1}^{-}$.

Consideremos los dos subcasos.

CASO 2 ii a) Si $v_{2} \in L_{1}^{+}$, entonces $x, y \in L_{1}^{-} s\left(G_{2}^{-}\right)<s\left(G_{1}^{-}\right)=\ell-1$, es decir $s\left(G_{2}^{-}\right) \leq \ell-2=\left|L_{2}^{-}\right|-3$, y además $s\left(G_{2}^{+}\right)=s\left(G_{1}^{+}\right)=\ell-2$. Como $x, y$ pertenecen a $L_{1}^{-}$por lema 3.3.4, existe una recta en $\mathcal{C}\left(L_{1}\right)$ tal que su eje pertenece a $L_{1}^{-}$. Sea $L_{i}$ la primera recta en $C\left(L_{1}\right)$ tal que su eje 
$v_{i}$ está en $L_{1}^{-}$. Claramente $L_{1}^{+}=L_{2}^{+}=\ldots=L_{i-1}^{+}, v_{1}, v_{2}, \ldots, v_{i-1} \in L_{1}^{+}$ y $L_{i}$ intersecta al triángulo vacío $x y z$. Considere la recta $L\left(v_{i-1}, v_{i}\right)$ y observe que $\left|L^{-}\left(v_{i-1}, v_{i}\right)\right|=\left|L_{i-1}^{-}\right|$y $\left|L^{+}\left(v_{i-1}, v_{i}\right)\right|=\left|L_{i-1}^{+}\right|+1$, por lo tanto

$$
\begin{aligned}
s\left(G^{-}\left(v_{i-1}, v_{i}\right)\right) & =s\left(G_{i-1}^{-}\right) \leq s\left(G_{2}^{-}\right)=\ell-2=\left|L_{2}^{-}\right|-3=\left|L_{i-1}^{-}\right|-3 \\
& =\left|L^{-}\left(v_{i-1}, v_{i}\right)\right|-3 .
\end{aligned}
$$

Además

$$
\begin{aligned}
s\left(G^{+}\left(v_{i-1}, v_{i}\right)\right) & =s\left(G_{i-1}^{+}\right)=s\left(G_{2}^{+}\right)=\ell-2=\left|L_{2}^{+}\right|-2=\left|L_{i-1}^{+}\right|-2 \\
& =\left|L^{+}\left(v_{i-1}, v_{i}\right)\right|-3 .
\end{aligned}
$$

por lo tanto, por hipótesis de inducción, existen $T^{-}\left(v_{i-1}, v_{i}\right)$ y $T^{+}\left(v_{i-1}, v_{i}\right)$ árboles generadores planos de $\left(G^{-}\left(v_{i-1}, v_{i}\right)\right)^{c}$ y $\left(G^{+}\left(v_{i-1}, v_{i}\right)\right)^{c}$ respectivamente. Es claro que la unión de éstos dos árboles generadores planos contiene un árbol generador plano de $G^{c}$ y el teorema se sigue.

CASO 2 ii b) Si $v \in L_{1}^{-}$, la demostración es análoga al caso 2 ii a).

CASo 2 iii) Supongamos que $s\left(G_{1}^{-}\right)>\left|L_{1}^{-}\right|-3$ y $s\left(G_{1}^{+}\right) \leq\left|L_{1}^{+}\right|-3$. Si para toda recta $L_{j} \in \mathcal{C}\left(L_{1}\right)$, tenemos que $s\left(G_{j}^{-}\right)>\left|L_{j}^{-}\right|-3$ y $s\left(G_{j}^{+}\right) \leq\left|L_{j}^{+}\right|-3$, entonces en particular para $L_{m}$, la única recta paralela a $L_{1}$ en $\mathcal{C}\left(L_{1}\right)$, tenemos que $s\left(G_{m}^{-}\right)>\left|L_{m}^{-}\right|-3$ y $s\left(G_{m}^{+}\right) \leq\left|L_{m}^{+}\right|-3$ es decir $s\left(G_{m}^{-}\right) \geq$ $\left|L_{m}^{-}\right|-2$ y $s\left(G_{m}^{+}\right) \leq\left|L_{m}^{+}\right|-3$.

Como $\left|L_{1}^{-}\right|=\ell+1=\left|L_{m}^{-}\right|$, entonces $L_{m}$ se encuentra a la izquierda de $L_{1}$ y tiene orientación opuesta a $L_{1}$, ver figura 3.11 , por lo tanto

$$
s\left(G_{1}^{-}\right)+s\left(G_{m}^{-}\right) \leq s(G) .
$$




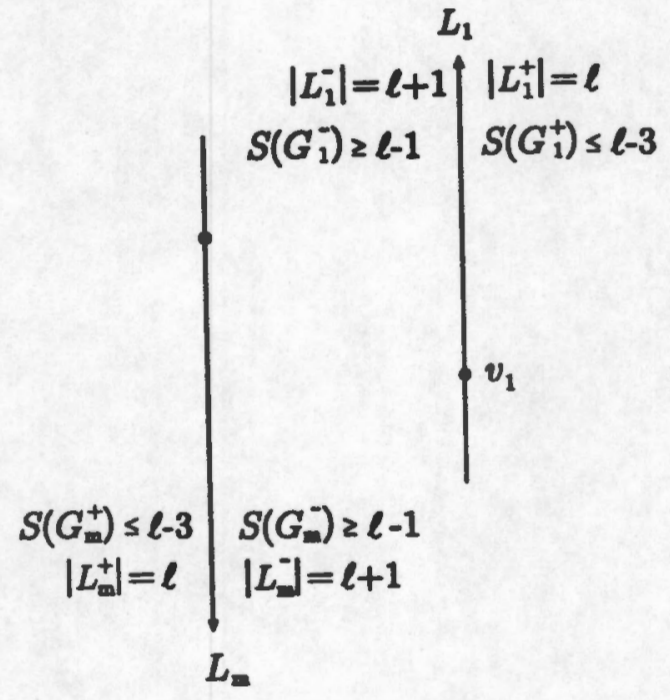

Figura 3.11

Como $s\left(G_{1}^{-}\right)>\left|L_{1}^{-}\right|-3$, entonces $s\left(G_{1}^{-}\right) \geq\left|L_{1}^{-}\right|-2$ por lo tanto

$$
s\left(G_{1}^{-}\right)+s\left(G_{m}^{-}\right) \geq\left|L_{1}^{-}\right|-2+\left|L_{m}^{-}\right|-2=2 \ell-2>s(G)
$$

lo cual no es posible. Luego, existe $L_{k} \in \mathcal{C}\left(L_{1}\right)$ tal que

$$
s\left(G_{k}^{-}\right) \leq\left|L_{k}^{-}\right|-3 \text { y } s\left(G_{k}^{+}\right) \leq\left|L_{k}^{+}\right|-3
$$

o bien

$$
s\left(G_{k}^{-}\right)>\left|L_{k}^{-}\right|-3 \text { y } s\left(G_{k}^{+}\right)>\left|L_{k}^{+}\right|-3 .
$$

En cualquiera de ambos casos el teorema se sigue, pues el primero es el caso 2 i) y el segundo es el caso 2 ii).

CASo $2 i v)$. Supóngase que $s\left(G_{1}^{-}\right) \leq\left|L_{1}^{-}\right|-3$ y $s\left(G_{1}^{+}\right)>\left|L_{1}^{+}\right|-3$. Si para cada recta $L_{j}$ de $\mathcal{C}\left(L_{1}\right)$ tenemos que

$$
s\left(G_{j}^{-}\right) \leq\left|L_{j}^{-}\right|-3 \quad \text { y } \quad s\left(G_{j}^{+}\right)>\left|L_{j}^{+}\right|-3 .
$$


Entonces en particular para $L_{m}$, la única recta paralela a $L_{1}$ en $\mathcal{C}\left(L_{1}\right)$, tenemos que

$$
s\left(G_{m}^{-}\right) \leq\left|L_{m}^{-}\right|-3 \text { y } s\left(G_{m}^{+}\right)>\left|L_{m}^{+}\right|-3
$$

Como $L_{m}$ se encuentra a la izquierda de $L_{1}$ y tiene orientación opuesta a $L_{1}$, se tiene que

$$
\left|L_{1}^{+}\right|-3<s\left(G_{1}^{+}\right) \leq s\left(G_{m}^{-}\right) \leq\left|L_{m}^{-}\right|-3
$$

y

$$
\left|L_{m}^{+}\right|-3<s\left(G_{m}^{+}\right) \leq s\left(G_{1}^{-}\right)=\left|L_{1}^{-}\right|-3
$$

Como

$$
\begin{array}{ll}
\left|L_{1}^{+}\right|=\ell, & \left|L_{1}^{-}\right|=\ell+1 \\
\left|L_{m}^{+}\right|=\ell, & \left|L_{m}^{-}\right|=\ell+1
\end{array}
$$

entonces

$$
s\left(G_{1}^{+}\right)=s\left(G_{1}^{-}\right)=s\left(G_{m}^{+}\right)=s\left(G_{m}^{-}\right)=\ell-2,
$$

por lo tanto, en $G_{m}^{-}$no existe un triángulo vacío tal que uno de sus vértices sea $v_{m}$, ni tampoco existe en $G_{1}^{-}$un triángulo vacío tal que uno de sus vértices sea $v_{1}$, ver figura 3.12 .

Sea $x y z$ un triángulo vacío de $G_{1}^{+}$. Como $L_{m}$ es paralela a $L_{1}$ y $L_{m}$ está a la izquierda de $L_{1}$, entonces el triángulo $x y z$ también es triángulo vacío de $G_{m}^{-}$, por lo tanto existe $j$, con $1<j<m$, tal que el triángulo $x y z$ está en $L_{j}^{-}$y consecuentemente en $G_{j}^{-}$y alguno de sus vértices es exactamente $v_{j}$, lo cual no es posible, pues usando el mismo razonamiento 


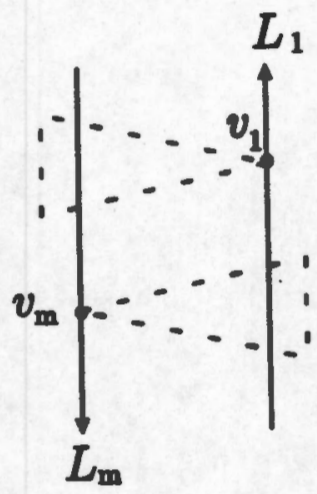

Figura 3.12

que se hizo para $L_{1}$ y $L_{m}$, en el caso de $L_{j}$ y su correspondiente recta paralela $L_{j}^{\prime}$, tenemos que no es posible que exista un triángulo vacío en $G_{j}^{-}$tal que $v_{j}$ sea uno de sus vértices, ver figura 3.13 .

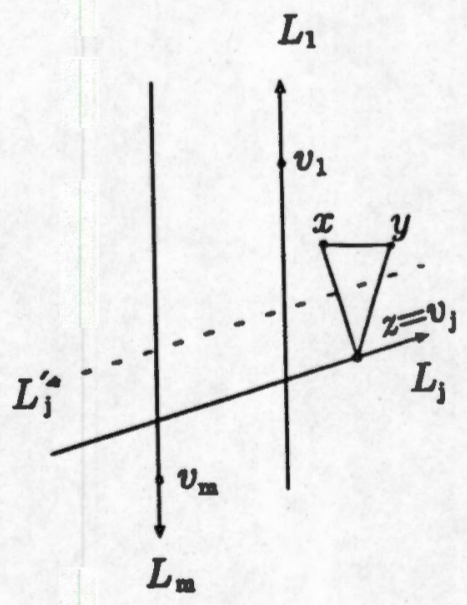

Figura 3.13

Por lo tanto existe $k, 1<k<m$ tal que

$$
s\left(G_{k}^{-}\right) \leq\left|L_{k}^{-}\right|-3 \quad \text { y } \quad s\left(G_{k}^{+}\right) \leq\left|L_{k}^{+}\right|-3
$$


o bien

$$
s\left(G_{k}^{-}\right)>\left|L_{k}^{-}\right|-3 \text { y } s\left(G_{k}^{+}\right)>\left|L_{k}^{+}\right|-3
$$

y es claro que en cualquiera de los dos casos se obtiene el teorema. 


\section{Referencias}

[1] Agarwal P.K, Pach J. Combinatorial Geometry, John Wiley \& Sons, Inc., New York, 1995.

[2] Avis D. y Fukuda K. Reverse search for enumeration. Discrete Applied Math. 6 (1996) 21-46.

[3] Behzad, Chartrand, Lesniak-Foster. Graphs \& Digraphs. Prindle, Weber \& Schmidt International Series. 1979.

[4] Bondy J.A., Murty U.S.R. Graph Theory with Applications. North Holland, 1981.

[5] Cummins R.L., Hamilton Circuits in tree graphs. IEEE. Trans. Circuit Theory 13 (1966), 82-90.

[6] Harary Frank. Graph Theory. Addison Wesley, 1969.

[7] Hernando C., Hurtado F., Márquez A., Mora M. y Noy M. Geometric tree graphs of points in convex position, Discrete Applied Math. 93 (1999), 51-66.

[8] Hernando Martín M. del Carmen. Complejidad de estructuras geométricas y combinatorias. Universitat Politécnica de Catalunya. 1999. Tesis Doctoral.

[9] Károli G., Pach J. \& Tóth G. Ramsey-type results for geometrics graphs I. Discrete and Computational Geometry, 18 (1997), 247-255. 
[10] Shank H. A note on Hamilton circuits in tree graphs. IEEE Trans. Circuit Theory 15 (1968), 86. 